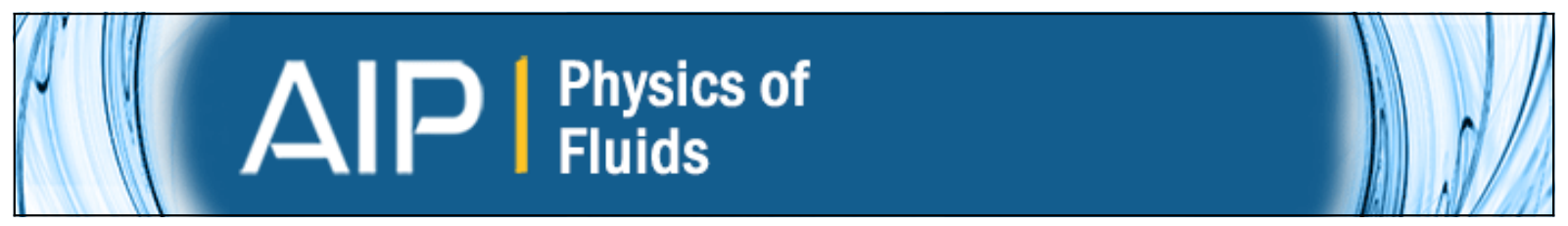

Linear stability analysis of miscible two-fluid flow in a channel with velocity slip at the walls

Sukhendu Ghosh, R. Usha, and Kirti Chandra Sahu

Citation: Physics of Fluids (1994-present) 26, 014107 (2014); doi: 10.1063/1.4862552

View online: http://dx.doi.org/10.1063/1.4862552

View Table of Contents: http://scitation.aip.org/content/aip/journal/pof2/26/1 ?ver=pdfcov

Published by the AIP Publishing

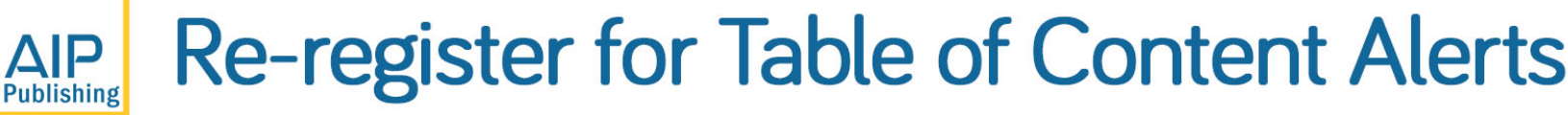




\title{
Linear stability analysis of miscible two-fluid flow in a channel with velocity slip at the walls
}

\author{
Sukhendu Ghosh, ${ }^{1}$ R. Usha, ${ }^{1, a)}$ and Kirti Chandra Sahu ${ }^{2}$ \\ ${ }^{1}$ Department of Mathematics, Indian Institute of Technology Madras, Chennai 600036, India \\ ${ }^{2}$ Department of Chemical Engineering, Indian Institute of Technology Hyderabad, \\ Yeddumailaram 502 205, Andhra Pradesh, India
}

(Received 20 August 2013; accepted 5 January 2014; published online 24 January 2014)

\begin{abstract}
The linear stability characteristics of pressure-driven miscible two-fluid flow with same density and varying viscosities in a channel with velocity slip at the wall are examined. A prominent feature of the instability is that only a band of wave numbers is unstable whatever the Reynolds number is, whereas shorter wavelengths and smaller wave numbers are observed to be stable. The stability characteristics are different from both the limiting cases of interface dominated flows and continuously stratified flows in a channel with velocity slip at the wall. The flow system is destabilizing when a more viscous fluid occupies the region closer to the wall with slip. For this configuration a new mode of instability, namely the overlap mode, appears for high mass diffusivity of the two fluids. This mode arises due to the overlap of critical layer of dominant instability with the mixed layer of varying viscosity. The critical layer contains a location in the flow domain at which the base flow velocity equals the phase speed of the most unstable disturbance. Such a mode also occurs in the corresponding flow in a rigid channel, but absent in either of the above limiting cases of flow in a channel with slip. The flow is unstable at low Reynolds numbers for a wide range of wave numbers for low mass diffusivity, mimicking the interfacial instability of the immiscible flows. A configuration with less viscous fluid adjacent to the wall is more stable at moderate miscibility and this is also in contrast with the result for the limiting case of interface dominated flows in a channel with slip, where the above configuration is more unstable. It is possible to achieve stabilization or destabilization of miscible two-fluid flow in a channel with wall slip by appropriately choosing the viscosity of the fluid layer adjacent to the wall. In addition, the velocity slip at the wall has a dual role in the stability of flow system and the trend is influenced by the location of the mixed layer, the location of more viscous fluid and the mass diffusivity of the two fluids. It is well known that creating a viscosity contrast in a particular way in a rigid channel delays the occurrence of turbulence in a rigid channel. The results of the present study show that the flow system can be either stabilized or destabilized by designing the walls of the channel as hydrophobic surfaces, modeled by velocity slip at the walls. The study provides another effective strategy to control the flow system. ( 2014 AIP Publishing LLC. [http://dx.doi.org/10.1063/1.4862552]
\end{abstract}

\section{INTRODUCTION}

The stability of a parallel two-fluid flow with viscosity stratification is relevant in numerous natural and industrial applications, such as the generation of water waves by wind, pipeline lubrication, air-water flow in nuclear reactor cooling towers, primary atomization of jets, and extrusion. There are different ways of achieving a viscosity stratification which include (i) considering immiscible fluids in contact with each other and in this case, there is a discontinuity in viscosity across a

a)Author to whom correspondence should be addressed. Electronic mail: ushar@iitm.ac.in 
sharp interface, (ii) varying continuously the temperature or concentration in which case a diffusive interface of nonzero thickness occurs, and (iii) using a non-Newtonian fluid. The instabilities arising due to viscosity stratification are discussed in detail in a recent review article by Govindarajan and Sahu. ${ }^{1}$ As mentioned in this article, apart from gaining knowledge about the nonlinear stages of growth and transition to turbulence, there is lot more to understand and principal questions to be addressed on the effect of viscosity stratification even in the linear regime. This motivates further studies to understand instability characteristics of viscosity stratified flows that can improve the performance of many industrial processes. The present study attempts to provide some information on the instability that arises in miscible three-layer channel flow with velocity slip at the walls.

$\mathrm{Yih}^{2}$ is the first to consider the stability of a Couette-Poiseuille flow in a rigid channel with sharp jump in viscosity. He focused on long waves and observed an interfacial mode of instability at any Reynolds number. Since then, there have been several studies addressing many aspects of this interfacial instability. ${ }^{3-12}$ The essence of these studies is that, in order to achieve a linearly stable flow, one should place the less viscous fluid in a thin layer close to the wall to stabilize long waves and provide enough interfacial tension to stabilize short waves.

There are also investigations in stratified flows in a rigid channel in which the fluid properties vary over the entire domain or at least over a large portion of it. ${ }^{13-15}$ Wall and Wilson ${ }^{15}$ studied the influence of continuous viscosity variation due to a temperature gradient in a rigid channel and showed that the Péclet number has little influence on the stability and that the base state viscosity influences the Tollmien-Schlichting (TS) mode. The experiments ${ }^{16-20}$ on miscible two-fluid flow in different geometries revealed interesting instabilities. At low miscibility, instabilities driven by viscosity stratified flow are observed to be qualitatively similar to those in immiscible fluids. ${ }^{16-18}$

The miscible two-fluid flow in which the fluid layers are separated by a finite-thickness mixed layer also falls under the class of viscous stratified flows and has been examined by Ranganathan and Govindarajan, ${ }^{16}$ Ern et al..${ }^{17}$ Govindarajan, ${ }^{18}$ and Sahu et al. ${ }^{19}$ Ern et al. ${ }^{17}$ considered the influence of diffusion and mixed layer thickness in the miscible two-fluid Couette flow with a high degree of stratification in the mixed layer. They showed that growth rate exhibits a non-monotonic behaviour with respect to diffusion and that flows at intermediate Péclet numbers are more unstable than those without diffusion, when the thickness of the mixed layer was not too large. Govindarajan and co-workers ${ }^{16,18}$ investigated the effects of a thin viscosity layer created by miscibility of two fluids of same density but different viscosity for symmetric flow through a rigid channel and examined the effects of diffusion. They identified a new mode of instability associated with the overlap of critical layer and the viscosity stratified layer. This mode was found to be very sensitive to the effects of diffusion when the viscosity stratified mixed layer and the critical layer overlap with each other. The flow was shown to become more stable or unstable depending on the viscosity ratio. Their results ${ }^{18}$ showed that the flow becomes unstable at Reynolds number much lower than that for the corresponding immiscible configuration. The stability properties were similar to that of the interfacial mode at low values of diffusivity, but the behaviour was qualitatively different for higher values of diffusivity. Recently, Talon and Meiburg ${ }^{20}$ investigated the linear stability of miscible viscosity-stratified plane Poiseuille flow in the Stokes flow regime. They demonstrated that instabilities develop due to the effects of diffusion. Further, they showed that at large wave numbers, the instability occurs even when the highly viscous layer is in the core of the pipe.

The above efforts were aimed at understanding the effects of a stratification of viscosity in laminar channel flows with rigid walls. There are many situations and important applications, such as lubrication, microfluidics, ${ }^{21,22}$ biological and technological drag reduction surfaces, ${ }^{23,24}$ highspeed rarefied flow, ${ }^{25,26}$ polymer melt, ${ }^{27}$ and drag reduction in microchannel flows ${ }^{28-30}$ where the velocity of a viscous fluid exhibits a tangential slip on the wall. It would be reasonable to think of slip in terms of a relation between slip velocity and the wall shear stress. The slip length is the equivalent local distance below the rigid surface where the no-slip condition at the surface can be satisfied if the flow field were extended linearly outside the physical domain. Measurements of boundary slip of Newtonian liquids have been the subject of recent research. The experimental predictions by Zhu and Granick ${ }^{21}$ and molecular dynamic simulations by Thompson and Troian ${ }^{22}$ suggest the possibility of slip at the solid boundary. The slip boundary conditions are applicable in the investigations of problems where fluids interact with solids at small length scales and this 
includes flows in microfluidics, porous media, and biological system, such as arterial flows. The Navier's concept of slip has been commonly used in many investigations, ${ }^{27}$ which states that the relative velocity of the fluid with respect to the wall (slip velocity) is proportional to the shear rate at the wall. Reports by a number of recent experiments on microscale flows driven by pressuregradient indicate an apparent break-down of the no-slip condition with slip lengths as large as micrometers. ${ }^{31-33}$ There are also investigations of pressure-driven fluid flow in channels that display results consistent with slip at the solid boundary. ${ }^{34}$ This suggests that it is relevant to consider the effects of slip on the linear stability of wall bounded shear flow of a fluid system in a channel with slippery walls. In the case of Poiseuille flow bounded by the walls at $y= \pm h$, the Navier slip conditions are described as $u+\beta_{1} u_{y}=0$ at $y=+h$ and $u-\beta_{2} u_{y}=0$ at $y=-h$ where $\beta_{1}, \beta_{2}$ are different slip coefficients at the wall boundaries.

In fact, the slip effects have been incorporated in plane Poiseuille flow of a single fluid with both symmetric and asymmetric slip conditions. Gersting ${ }^{35}$ reported that wall slip has a stabilizing effect on the flow dynamics. The study by Spille and Rauh ${ }^{36}$ confirms the above conclusion. The results of $\mathrm{Gan}$ and $\mathrm{Wu}^{37}$ indicate that the wall slip causes short wave instability while the slip-flow model is stable for long waves. Lauga and $\operatorname{Cossu}^{38}$ formulated the linear stability of plane Poiseuille flow with different slip coefficients $\beta_{1}, \beta_{2}\left(\beta_{1} \neq \beta_{2}\right)$ at the wall boundaries but focused on the cases of symmetric slip $\left(\beta_{1}=\beta_{2}=\beta\right)$ and asymmetric slip $\left(\beta_{1}=\beta, \beta_{2}=0\right)$. Such differences in the wall slip can arise when the surface wettability, surface chemistry, and surface roughness at the walls of the channel are distinct. ${ }^{39}$ Their results showed that the presence of slip at the wall significantly increases the critical Reynolds number for linear stability. Ling et al. ${ }^{40}$ extended the study by Lauga and $\operatorname{Cossu}^{38}$ by considering an asymmetric slip boundary condition at the walls with $\beta_{1}, \beta_{2} \neq 0$. They found that depending on the slip length, the slip plays a dual role by either stabilizing or destabilizing the flow system, depending on the slip length. For the symmetric slip boundary conditions, their results showed a similar stabilizing trend of Lauga and $\mathrm{Cossu}^{38}$ for $\beta>0.0011$. However, for $\beta<0.0011$ the slip has a destabilizing effect. A similar behaviour is observed for asymmetric case also. In the case of microflows mentioned above, the amount of slip at the wall is linearly proportional to the gradient of the tangential velocity at the wall, with proportionality coefficient defined as the slip length..$^{25,41}$ If the slip wall conditions are used, the Navier-Stokes equations are valid for slip length up to $0.1 .^{41}$

Motivated by the results of Lauga and Cossu, ${ }^{38}$ Sahu et al. ${ }^{42}$ analyzed the relative rolls of angle of divergence and velocity slip in the linear stability of a diverging channel flow. Using the Maxwell velocity slip boundary conditions ${ }^{43}$ at the walls, they showed that unlike the flow in a straight channel, wall slip has a destabilizing influence in flow through diverging channel at low Knudsen numbers $(K n)$. The Maxwell slip boundary conditions ${ }^{43}$ are given by $u \pm K n \partial u / \partial y=0$ at $y= \pm h$, where $K n$ is the ratio of slip length to the local half-width of the channel. As in the previous investigations, ${ }^{37,38,44,45}$ they also considered $K n$ less than 0.1 .

You and Zheng ${ }^{46}$ investigated the effects of boundary slip on the stability of viscosity-stratified microchannel flow in which two immiscible fluids are separated by a sharp interface. Their results revealed that the stability of stratified microchannel flow is enhanced by boundary slip. The slip effects were observed to be strong at small and large viscosity contrasts and were relatively weak when viscosity contrast is close to one.

A linear stability analysis of pressure-driven flow in a plane channel with slip at the boundary examined by Webber, ${ }^{47}$ in the presence of temperature variation is analogous to that of Wall and Wilson ${ }^{14}$ in a rigid channel. Taking viscosity dependence on temperature to be linear, they concluded that boundary slip is linearly stabilizing. For a fixed value of slip parameter, an increase in temperature enhanced stability; but the critical Reynolds number decreases and then increases with temperature.

The aim of the present study is to examine the linear stability of a symmetric Poiseuille flow of two miscible fluids of equal densities and different viscosities (say $\mu_{1}$ and $\mu_{2}$ ) separated by a mixed layer (in which viscosity varies continuously from $\mu_{1}$ to $\mu_{2}$ ) through a channel with velocity slip at the walls. The analysis is restricted to symmetric slip cases. The results for the flow system in a rigid channel are recovered in the limit $\beta=0$. It extends the investigations by Ern et al. ${ }^{17}$ Govindarajan, ${ }^{18}$ and Sahu et al. ${ }^{19}$ for a miscible two-fluid flow in a rigid channel, where the effects of a continuous variation of concentration across a layer subject to diffusion have been studied. To 
the best of our knowledge, the present study is a first attempt to clarify the above effects in a channel with velocity slip at the walls.

In view of the discussion and the available investigations in the literature on flow in a channel with slip at the walls, the study can be thought of as describing the effects of a hydrophobic surface on stability in wall bounded viscosity-stratified flow, where the hydrophobic surface is represented by a slip boundary condition on the surface ${ }^{44}$ and the velocity of the fluid exhibits a tangential slip on the walls. The results generated can be used according to the applications for which they are relevant. For example, if a PDMS (Polydimethylsiloxane) channel is hosting an oil-water flow, then two possible configurations can be considered for the stability analysis by using the formulation in the present study (see Sec. II A). In the first configuration, water flows adjacent to the hydrophobic wall and oil flows in the core region of the channel. As a result, the contact angle will be high (more than $90^{\circ}$ and up to $150^{\circ}$ ) and the surface energy will be less. Then the effect of the wall slip to reduce wall shear is more, which in turn stabilizes the flow system. Note that in the first configuration, fluid with lower viscosity (water) is adjacent to the hydrophobic channel wall. In the second configuration, oil (more viscous fluid) flows adjacent to hydrophobic wall, thus the contact angle will be less and the surface energy will be high. Therefore, the effect of wall slip is less to reduce wall shear. This causes the flow system to be more unstable. The above conclusions are confirmed by the analysis/results presented in Sec. III.

The paper is organized as follows. The mathematical formulation of the base state and the linear stability analysis are presented in Sec. II. The results of the stability analysis are discussed in Sec. III. The conclusions are presented in Sec. IV.

\section{MATHEMATICAL FORMULATION}

\section{A. Governing equations}

The linear stability of a pressure-driven laminar two-dimensional flow of two miscible, Newtonian, incompressible fluids in a horizontal plane channel with wall slip is considered. The two fluids have the same density $\rho$ but different viscosities. They are separated by a mixed layer of viscositystratified fluid. The flow is symmetric about the centerline of the channel, thus the formulation is presented for the upper half of the channel (see Fig. 1). A Cartesian coordinate system is chosen with $x$ and $y$ directions along and perpendicular to the centerline of the channel $(y=0)$. The walls of the channel are located at $y= \pm H$. The fluids of viscosities $\mu_{1}$ and $\mu_{2}$ occupy the regions $0 \leq y$ $\leq h$ and $h+q \leq y \leq H$, respectively. There is a thin layer where the two fluids mix-up and a local stratification of viscosity is created. This layer is referred to as a mixed layer of thickness $q$ and it occupies the region $h \leq y \leq h+q$. The downstream growth of the mixed layer thickness is neglected under the assumption that the two fluids diffuse into each other very slowly (i.e., the Péclet number, defined later in this section, is high; also see Appendices A and B). The basic viscosity is related to the basic concentration profile and it varies monotonically between the two fluids with viscosity

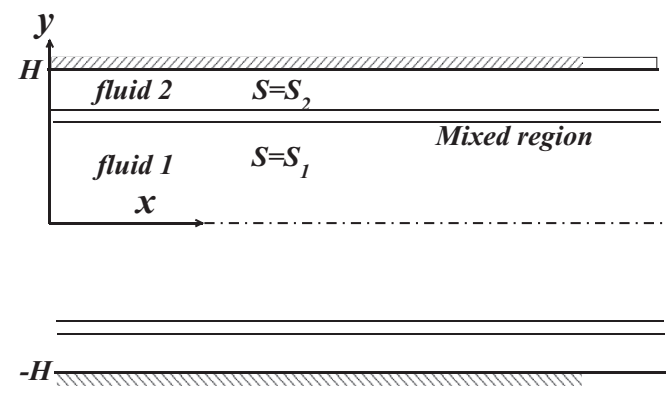

FIG. 1. Schematic of the flow system considered. The core and annular regions of the slippery channel contain the fluids "1" and " 2 ," respectively. Here fluid " 1 " occupies the region $-h \leq y \leq h$ and both the fluids are separated by a mixed layer of uniform thickness $q$. The slippery walls of the channel are located at $y= \pm H$. 
$\mu_{1}$ (viscosity of fluid-1) and $\mu_{2}$ (viscosity of fluid-2) in the mixed layer. In the present study, an exponential dependence of the viscosity $\mu$ on the concentration is assumed ${ }^{48}$ and is given by

$$
\mu=\mu_{1} \exp \left[R_{s}\left(\frac{S-S_{1}}{S_{2}-S_{1}}\right)\right]
$$

where $R_{s}=\left(S_{2}-S_{1}\right) \frac{\mathrm{d}}{\mathrm{d} s}(\ln \mu)$ is the log-mobility ratio of the scalar $S$. The scalar $S$ (can also be temperature) takes the values $S_{1}$ and $S_{2}$ in the regions $0 \leq y \leq h$ and $h+q \leq y \leq H$, respectively. This defines the basic viscosity as follows:

$$
\mu=\left\{\begin{array}{ll}
\mu_{1} & \text { if } 0 \leq y \leq h \\
\mu_{m}(y) & \text { if } h \leq y \leq h+q \\
\mu_{2} & \text { if } h+q \leq y \leq H
\end{array},\right.
$$

where $\mu_{2}=\mu_{1} \mathrm{e}^{R_{s}}$ and $\mu_{m}(y)=\mu_{1} \mathrm{e}^{R_{s}\left(\frac{S-S_{1}}{S_{2}-S_{1}}\right)}$. The governing equations are the continuity and the Navier-Stokes equations together with a scalar-transport equation for the concentration of the scalar. The boundary conditions are the symmetry condition at the centerline and the velocity slip condition at the channel wall, which are given by

$$
\begin{gathered}
\frac{\partial u}{\partial y}=0, \quad v=0 \quad \text { at } y=0, \\
u=-\beta_{1} \frac{\partial u}{\partial y}, \quad v=0 \quad \text { at } y=H .
\end{gathered}
$$

Here $(u, v)$ are the components of the velocity along the $x$ and $y$ directions, respectively, and $\beta_{1}$ is the dimensional slip parameter. The equations and the boundary conditions governing the flow dynamics are made dimensionless by using the following scales:

$$
\begin{array}{r}
x^{*}=\frac{x}{H}, \quad y^{*}=\frac{y}{H}, \quad t^{*}=\frac{Q}{H^{2}} t, \quad\left(u^{*}, v^{*}\right)=\frac{H}{Q}(u, v), \quad p^{*}=\frac{H^{2}}{\rho Q^{2}} p, \\
\mu^{*}=\frac{\mu}{\mu_{1}}, \quad h^{*}=\frac{h}{H}, \quad q^{*}=\frac{q}{H}, \quad m=\frac{\mu_{2}}{\mu_{1}}, \quad \beta=\frac{\beta_{1}}{H}, \quad S^{*}=\frac{S-S_{1}}{S_{2}-S_{1}}, \quad \mu_{m}^{*}=\frac{\mu_{m}(y)}{\mu_{1}},
\end{array}
$$

where $Q$ is the total volume flow rate per unit distance in the spanwise direction, $p$ is pressure, and $t$ is time. They are given by (after suppressing $*$ )

$$
\begin{gathered}
u_{x}+v_{y}=0, \\
u_{t}+u u_{x}+v u_{y}=\frac{\partial}{\partial x}\left[-p+\frac{2}{R e} \mu u_{x}\right]+\frac{\partial}{\partial y}\left[\frac{1}{R e} \mu\left(u_{y}+v_{x}\right)\right], \\
v_{t}+u v_{x}+v v_{y}=\frac{\partial}{\partial x}\left[\frac{1}{R e} \mu\left(u_{y}+v_{x}\right)\right]+\frac{\partial}{\partial y}\left[-p+\frac{2}{R e} \mu u_{y}\right], \\
s_{t}+u s_{x}+v s_{y}=\frac{1}{P e}\left[s_{x x}+s_{y y}\right], \\
u_{y}=0, \quad v=0 \quad \text { at } \quad y=0, \\
u=-\beta u_{y}, \quad v=0 \quad \text { at } \quad y=1,
\end{gathered}
$$

where $P e=Q / \mathcal{D}$ is the Péclet number, $\mathcal{D}$ is the mass diffusivity, $R e=\rho Q / \mu_{1}$ is the Reynolds number, and $S c=P e / R e$ is the Schmidt number. 


\section{B. Base state}

The base state is obtained by solving Eqs. (6)-(9) along with the boundary conditions (10) and (11) by assuming a steady, parallel, fully developed flow:

$$
\operatorname{Re}\left(\frac{d P_{B}}{d x}\right)=\frac{d}{d y}\left[\mu_{B}(y) \frac{d U_{B}(y)}{d y}\right] .
$$

The solution of the above equation is given by

$$
U_{B}(y)= \begin{cases}\frac{G}{2}\left[y^{2}-h^{2}+\frac{(h+q)^{2}-1-2 \beta}{m}-2 \int_{h}^{h+q} \frac{y}{\mu_{m}(y)} d y\right] & \text { if } 0 \leq y \leq h \\ \frac{G}{2}\left[\frac{(h+q)^{2}-1-2 \beta}{m}-2 \int_{y}^{h+q} \frac{y}{\mu_{m}(y)} d y\right] & \text { if } h \leq y \leq h+q \\ \frac{G}{2 m}\left(y^{2}-1-2 \beta\right) & \text { if } h+q \leq y \leq 1\end{cases}
$$

where $G=R e P_{B x}$ and

$$
\mu_{B}(y)= \begin{cases}1 & \text { if } 0 \leq y \leq h \\ \mu_{m}(y)=\mathrm{e}^{R_{s} s_{B}(y)} & \text { if } h \leq y \leq h+q . \\ m=\mathrm{e}^{R_{s}} & \text { if } h+q \leq y \leq 1\end{cases}
$$

Here, the subscript $B$ designates the base state variables and $s_{B}$ is taken to be a fifth order polynomial in the mixed layer ${ }^{48}$ such that the concentration profile is smooth up to the second derivative at $y=$ $h$ and $y=h+q$ :

$$
s_{B}(y)= \begin{cases}0 & \text { if } 0 \leq y \leq h \\ \sum_{i=1}^{6} a_{i} y^{i-1} & \text { if } h \leq y \leq h+q, \\ 1 & \text { if } h+q \leq y \leq 1\end{cases}
$$

where $a_{i}, i=1,2, \ldots, 6$, are given by

$$
\begin{aligned}
& a_{1}=-\frac{h^{3}}{q^{5}}\left(6 h^{2}+15 h q+10 q^{2}\right), \quad a_{2}=\frac{30 h^{2}}{q^{5}}(h+q)^{2}, \\
& a_{3}=-\frac{30 h}{q^{5}}(h+q)(2 h+q), \quad a_{4}=\frac{10}{q^{5}}\left(6 h^{2}+6 h q+q^{2}\right), \\
& a_{5}=-\frac{15}{q^{5}}(2 h+q), \quad a_{6}=\frac{6}{q^{5}} .
\end{aligned}
$$

The dimensionless pressure-gradient is determined by requiring that $\int_{0}^{1} U_{B}(y) d y=1$. Note that $m$ $>1$ and $m<1$ represent situations when the highly viscous fluid is adjacent to the wall and core of the channel, respectively; $m=1$ represents the situation without viscosity stratification. Also $m$ $=1.2,1$, and 0.8 correspond to $R_{s}=0.1823,0$, and -0.2231 , respectively. The thickness of the mixed layer, $q$, is 0.1 . The value has been kept constant in this study. However, halving the value of $q$ does not change the result qualitatively. The profiles of the typical base state velocity $U_{B}(y)$ and viscosity $\mu_{B}(y)$ are presented in Fig. 2 for different values of $m$. The base state velocity $U_{B}(y)$ satisfies $U_{B}(y)=\beta \frac{\partial U_{B}(y)}{\partial y}$ at $y=1$ and $\frac{\partial U_{B}(y)}{\partial y}=0$ at $y=0$. Figs. 2(a) and 2(b) present the base state velocity and viscosity profiles for $\beta=0$ and $\beta=0.1$, respectively, when $h=0.7$ with the mixed layer located close to the channel wall. It can be seen that the centerline velocity is more when the highly viscous fluid is close to the rigid wall of the channel than that when a less viscous fluid is close to the rigid channel wall for $\beta=0$ (see Fig. 2(a)). The same trend is observed for a channel with velocity slip at the wall, i.e., for example, $\beta=0.1$ (see Fig. 2(b)). Close inspection also reveals that the centerline velocity in a channel with wall slip is less as compared to that in a rigid channel for any viscosity contrast. 
(a)

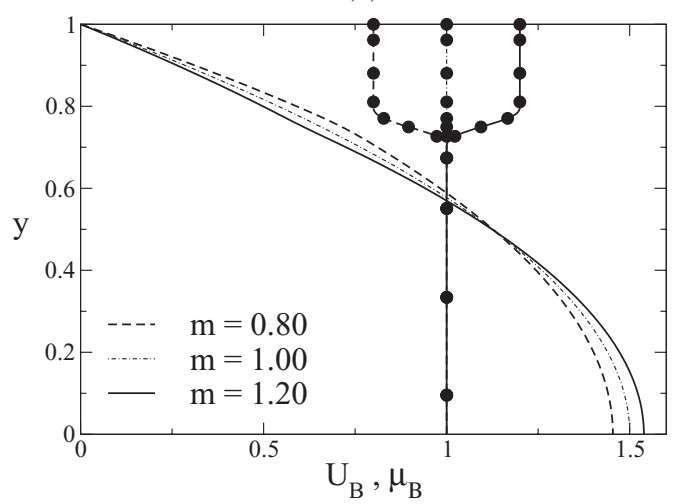

(c)

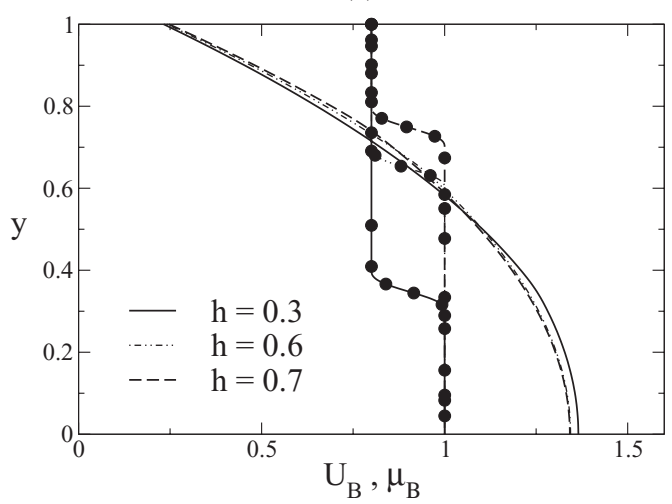

(e)

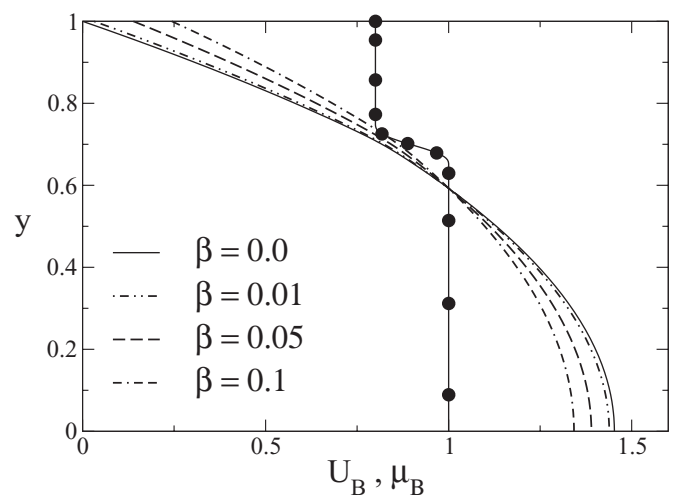

(b)

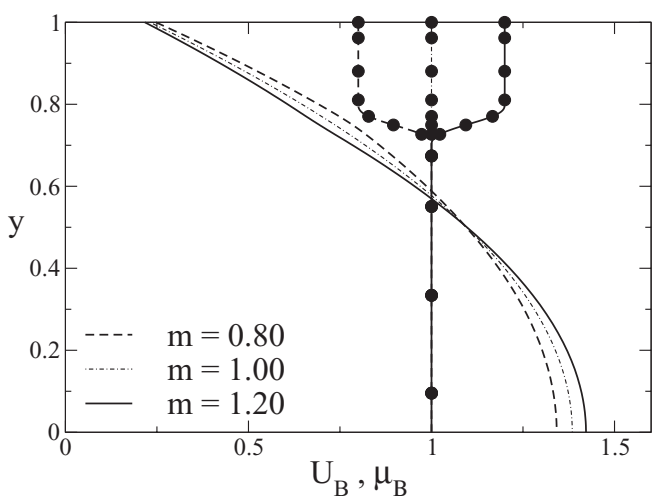

(d)

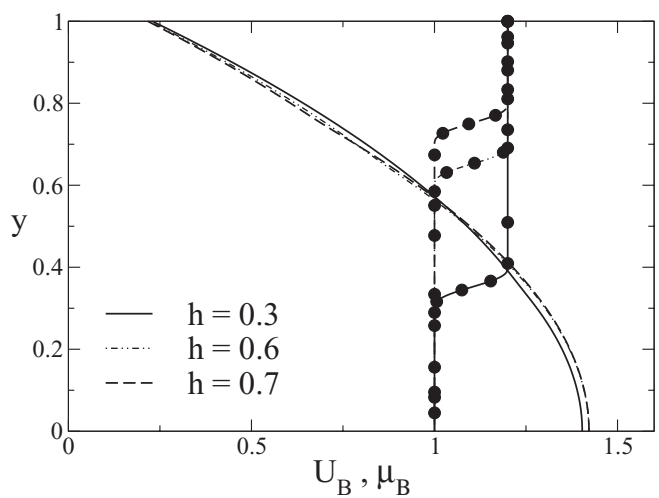

(f)

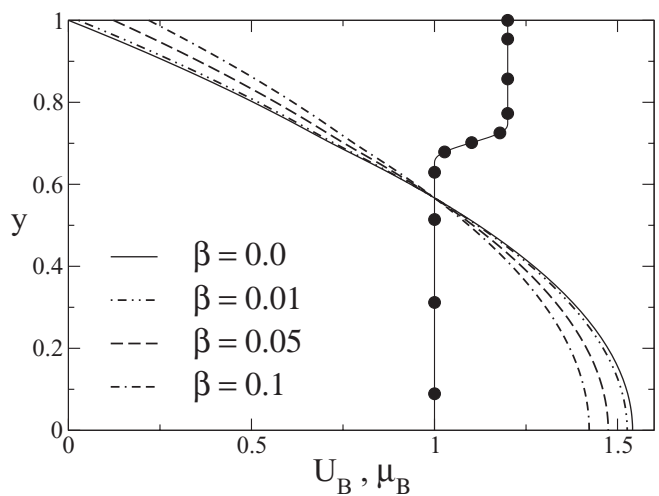

FIG. 2. Base state velocity and viscosity (with circles) profiles. Influence of viscosity stratification ( $m$ ) for $h=0.7$ : (a) $\beta=0$; (b) $\beta=0.1$. Influence of location (h) of the mixed layer for $\beta=0.1$ : (c) $m=0.8$; (d) $m=1$.2. Influence of slip parameter $\beta$ for $h=0.65$ : (e) $m=0.8$; (f) $m=1.2$. The value of $q$ is 0.1 in all the panels.

The slip parameter values used in the present study ( $\beta$ ranging from 0.01 to 0.1 ) are the same as those in the investigations ${ }^{35-38,44,46}$ of a single fluid or immiscible two-fluid flow in a channel with velocity slip at the wall. In these studies, the slip parameter values are based on the review on the experimental investigations by Lauga et al. ${ }^{49}$ This range of $\beta$ can be realized for a flow in a hydrophobic channel of height ranging from $0.8 \mu \mathrm{m}(40 \mu \mathrm{m})$ to $4 \mu \mathrm{m}(200 \mu \mathrm{m})$, and corresponds to a slip length of $20 \mathrm{~nm}(40 \mathrm{~nm})$.

Figs. 2(c) and 2(d) present the influence of the location of the mixed layer on the base state velocity and the viscosity profiles for $m=0.8$ and $m=1.2$, respectively, for $\beta=0.1$. It can be seen that decreasing the value of $h$ (moving the mixed layer away from the wall) decreases the centerline 
velocity when the less viscous fluid is adjacent to the wall $(m=0.8)$. However, the opposite trend is observed when the highly viscous fluid is adjacent to the wall $(m=1.2)$. The effects of $\beta$ on the base state velocity and viscosity profiles are shown in Figs. 2(e) and 2(f) for $m=0.8$ and $m=1.2$, respectively. It can be observed that increasing the velocity slip at the wall decreases the centerline velocity for both the viscosity ratios considered. It can also be observed that the centerline velocity for $m=0.8$ is smaller than that for $m=1.2$ for each value of $\beta$. Further, a slip at the wall reduces the wall shear.

\section{Linear stability analysis}

The temporal evolution of the base flow $\left(U_{B}(y), \mu_{B}(y), P_{B}(x)\right)$ described by Eqs. (13)-(16) is examined using linear stability analysis. The flow variables are split into the base state quantities and two-dimensional perturbations (designated by a hat) as

$$
(u, v, p, s)=\left(U_{B}(y), 0, P_{B}(x), s_{B}(y)\right)+(\hat{u}, \hat{v}, \hat{p}, \hat{s})(y) \mathrm{e}^{\mathrm{i}(\alpha x-\omega t)},
$$

where $\mathrm{i} \equiv \sqrt{-1}, \alpha$ is the streamwise disturbance wave number, $\omega=\alpha c$ is the frequency of the twodimensional disturbance, and $c$ is the complex phase speed. The flow is linearly unstable if $\operatorname{Im}(\omega)=$ $\omega_{i}>0$, stable if $\operatorname{Im}(\omega)=\omega_{i}<0$, and neutrally stable if $\operatorname{Im}(\omega)=\omega_{i}=0$. It is to be noted that the perturbation viscosity $\hat{\mu}$ is given by $\hat{\mu}=\frac{\partial \mu_{B}}{\partial s_{B}} \hat{s}$. The velocity perturbations are expressed in terms of the stream function perturbation $\hat{\phi}$ as $(\hat{u}, \hat{v})=\left(\hat{\phi_{y}},-\hat{\phi_{x}}\right)$. Modified Orr-Sommerfeld system is then derived from the non-dimensional governing equations (6)-(9) and the boundary conditions (10)-(13) using a standard procedure ${ }^{50}$ and are given by (after suppressing hat $\left(^{\wedge}\right)$ symbols)

$$
\begin{gathered}
\mathrm{i} \alpha \operatorname{Re}\left[\phi^{\prime \prime}\left(U_{B}-c\right)-\alpha^{2} \phi\left(U_{B}-c\right)-U_{B}{ }^{\prime \prime} \phi\right]=\mu_{B} \phi^{\prime \prime \prime \prime}+2 \mu_{B}{ }^{\prime \prime \prime \prime}+\left(\mu_{B}^{\prime \prime}-2 \alpha^{2} \mu_{B}\right) \phi^{\prime \prime}- \\
2 \alpha^{2} \mu_{B}{ }^{\prime}+\left(\alpha^{2} \mu_{B}{ }^{\prime \prime}+\alpha^{4} \mu_{B}\right) \phi+U_{B}{ }^{\prime \prime \prime}+2 U_{B}{ }^{\prime \prime} \mu^{\prime}+\left(U_{B}^{\prime \prime \prime}+\alpha^{2} U_{B}{ }^{\prime}\right) \mu, \\
\mathrm{i} \alpha \operatorname{Pe}\left[\left(U_{B}-c\right) s-s_{B}{ }^{\prime} \phi\right]=\left(s^{\prime \prime}-\alpha^{2} s\right), \\
\phi^{\prime}=-\beta \phi^{\prime \prime}, \quad \phi=s=0 \quad \text { at } \quad y=1, \\
\phi^{\prime}=\phi^{\prime \prime \prime}=s^{\prime}=0 \quad \text { at } \quad y=0 \quad \text { (sinuous mode), }
\end{gathered}
$$

where prime (') denotes differentiation with respect to $y$. The above equations incorporate the terms that arise due to the continuous variations of the base flow velocity and viscosity perturbations. Equations (18)-(21) constitute an eigenvalue problem and determine the linear stability of infinitesimal two-dimensional disturbances of the miscible three-layer pressure-driven flow in a channel with wall slip. The classical Orr-Sommerfeld equation is recovered ${ }^{50}$ for constant viscosity case. The modified Orr-Sommerfeld system is numerically solved by using Chebyshev spectral collocation method (Canuto et al. ${ }^{51}$ ) and the public domain software, LAPACK. The results are presented for sinuous mode (described by Eq. (21) at the centerline of the channel) as it was observed to be the dominant mode for the range of parameters considered. A large number of grid points are taken in the mixed layer, since the gradients are large in this layer. This is achieved by using the stretching function (Govindarajan ${ }^{18}$ )

$$
y_{j}=\frac{a}{\sinh \left(b y_{0}\right)}\left[\sinh \left\{\left(y_{c}-y_{0}\right) b\right\}+\sinh \left(b y_{0}\right)\right],
$$

where $y_{j}$ are the locations of the grid points, $a$ is the mid point of the mixed layer, and $y_{c}$ is a Chebyshev collocation point, given by

$$
y_{c}=0.5\left\{\cos \left[\pi \frac{(j-1)}{(n-1)}\right]+1\right\}
$$


and

$$
y_{0}=\frac{1}{2 b} \ln \left[\frac{1+\left(\mathrm{e}^{b}-1\right) a}{1+\left(\mathrm{e}^{-b}-1\right) a}\right],
$$

where $n$ is the number of collocation points and $b$ is the degree of clustering. In the present study, the computations are performed with $b=8$ and using 121 collocation points. This gives an accuracy of at least five decimal places in the range of parameters considered.

\section{RESULTS}

In this section, the effects of location of the mixed layer, velocity slip at the walls, and level of diffusivity on the stability properties of the flow system are examined. The correctness and accuracy of the developed numerical code are first assessed by examining the neutral stability curves for single fluid channel flow with rigid walls and walls with slip (Fig. 3). The computations are performed for sinuous mode at the centerline $y=0$ with $m=1$ both for $\beta=0$ (rigid wall) and $\beta \neq 0$ (walls with slip). We found that the critical Reynolds number, $\operatorname{Re}_{c r}$, for $\beta=0$ is 3848.16 and it is to be noted that in the present study, the Reynolds number is based on the mass flux $Q$. The critical Reynolds number based on the maximum velocity is 5772.2 (Drazin and Reid ${ }^{50}$ ) and two thirds of this is the critical Reynolds number obtained in the present case, for $\beta=0$. The results show an excellent agreement with the available results for $\beta=0$. In the case of wall-slip $(\beta \neq 0)$, the neutral stability boundaries are shifted towards larger values of Reynolds number as compared to that for $\beta=0$ (see Fig. 3(a)). The critical Reynolds number $\left(R e_{c r}\right)$ as a function of $\beta$ is presented in Fig. 3(b). In view of the choice of characteristic velocity scale as maximum velocity by Lauga and Cossu, ${ }^{38} \boldsymbol{R e}_{c r}$ in the present study (Fig. 3(b)) is $\frac{2}{3}\left(1+3 \beta\right.$ ) times that obtained by Lauga and Cossu, ${ }^{38}$ for $\beta \neq 0$. Figs. 3(a) and 3(b) reveal the stabilizing effect of velocity slip at the wall.

Further, the computations are carried out and stability boundaries are obtained for the problem analyzed by Govindarajan, ${ }^{18}$ where the effects of miscibility on the linear stability of two-fluid channel have been examined by taking the walls of the channels to be rigid and imposing no slip condition. In the investigation by Govindarajan, ${ }^{18}$ the mean viscosity is taken to be a fifth-order polynomial whereas in the present study, it is assumed to vary exponentially. The results are obtained independently for the above two choices of viscosity profiles. The stability boundaries obtained with the latter choice of viscosity profile are presented in Figs. 4(a), 4(b), and 4(c) for $q=0.1, S c=0, m$ $=1.2$ and are observed to be qualitatively similar to those presented in Figure 2 of Govindarajan. ${ }^{18}$ It is to be noted that in her result the stability boundaries are presented in $\alpha-R_{a v}$ plane, where $R_{a v}$ is the Reynolds number based on the spatially averaged viscosity across the channel with rigid walls.

In Fig. 4(a), for small $h(h=0.2)$, there is a TS mode instability similar to that observed for a flow of a single fluid in a planar rigid channel. As the location of the mixed layer moves closer to the

(a)

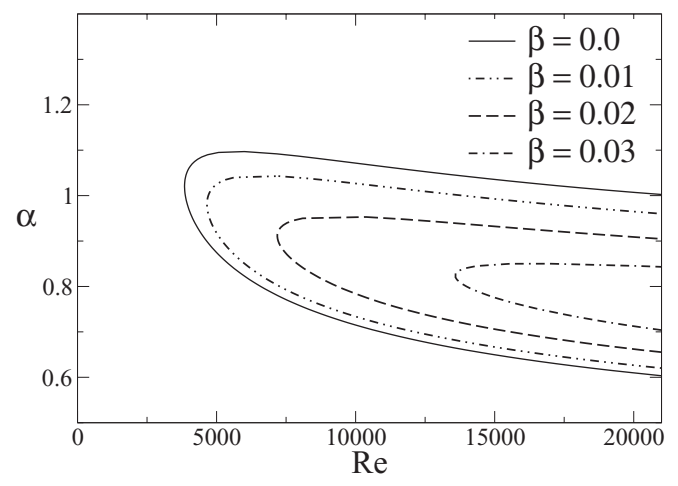

(b)

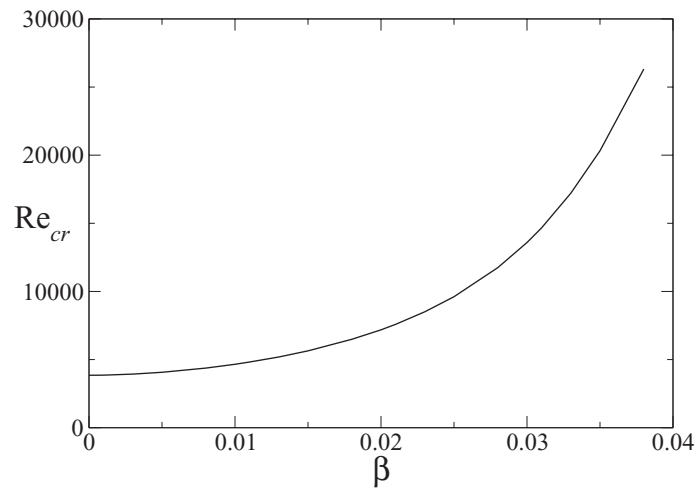

FIG. 3. (a) The neutral stability boundaries for a single fluid flow in a channel with no-slip $(\beta=0)$ and slip $\beta \neq 0$. (b) Critical Reynolds number as a function of slip parameter $\beta$. 
(a)

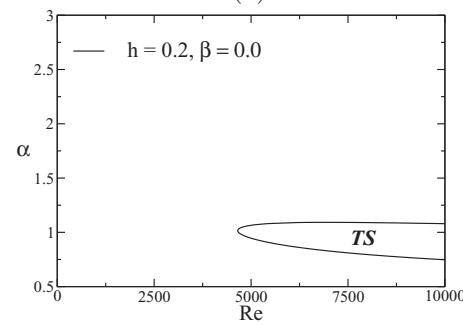

(b)

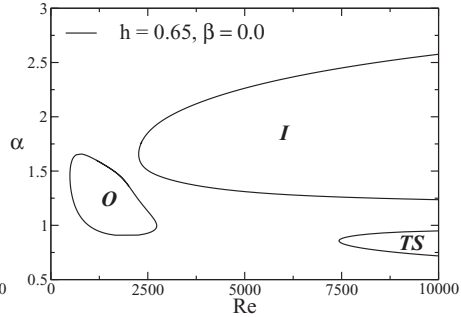

(c)

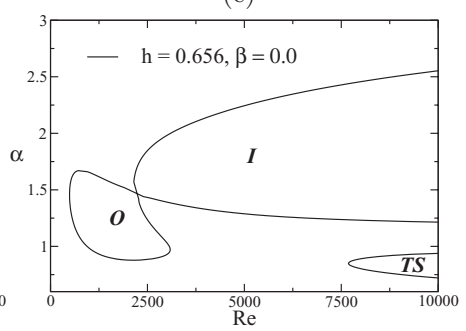

FIG. 4. ((a)-(c)) The neutral stability boundaries for $q=0.1, S c=0$, and $m=1.2$ for miscible two-fluid flow in a rigid channel.

rigid wall (Fig. 4(b), $h=0.65$ ), three modes of instability occupying distinct and sizable regions of $\alpha-R e$ plane are observed. They are referred to as the TS mode, the "I" or inviscid mode appearing at shorter wavelengths and the "O" or the overlap mode that becomes unstable at low Reynolds number. The "O" mode of instability arises due to the overlap of the critical layer of dominant instability with the mixed layer of varying viscosity, where the critical layer associated with a particular disturbance eigenmode is one that contains a critical location at which the base flow velocity equals the phase speed. In Fig. 4(b), the location of the critical layer $(y=0.7)$ overlaps the mixed region of the fluids $(0.65 \leq y \leq 0.75$ ). At $h=0.656$ (Fig. 4(c)), the "O" mode and the "I" mode are seen to be merged and the stability loop contains a bifurcation point. As the location of the mixed region goes further closer to the wall, all the three modes of instability coalesce and there is a large region of instability (see $\beta$ $=0$ curve in Fig. $7(\mathrm{f})$ ). The agreement with the available results ${ }^{18,38,50}$ gives sufficient confidence in using the code for further study and the results for the present study are furnished below. The results presented for $S c=0$ help us to compare the instabilities in miscible two-fluid flow in a channel with slippery wall with the available result in a rigid channel. ${ }^{18}$ In addition, the computations are carried out for $S c>0$ to understand the effects of diffusion.

The eigenspectra for $R e=400$ is shown in Fig. 5(a) (for $\beta=0.0,0.01$ ) and Fig. 5(b) (for $\beta=$ $0.0,0.05$ ), respectively, for $h=0.2$ and 0.65 , with the other parameters as $\alpha=1.35, q=0.1$, and $S c=1$. When $h=0.2$, the mixed layer and the critical layer of the dominant disturbance are well separated. Figs. 5(a) and 5(b) show that the growth rate $\left(\omega_{i}=\alpha c_{i}\right)$ is negative and the two-fluid flow in both the rigid channel and the channel with wall slip is stable. When $h=0.65$, the two layers overlap. In this case, the growth rate of the disturbance is positive for two-fluid miscible flow in a rigid channel $(\beta=0$; Figs. 5(a) and 5(b)). At this $\operatorname{Re}(=400)$, this is not so for two-fluid channel flow with wall slip. For $\beta=0.01$, the growth rate is positive (Fig. 5(a)) while for $\beta=0.05$, it is negative (Fig. 5(b)). The growth rate is more for flow in a rigid channel than in a channel with wall

(a)

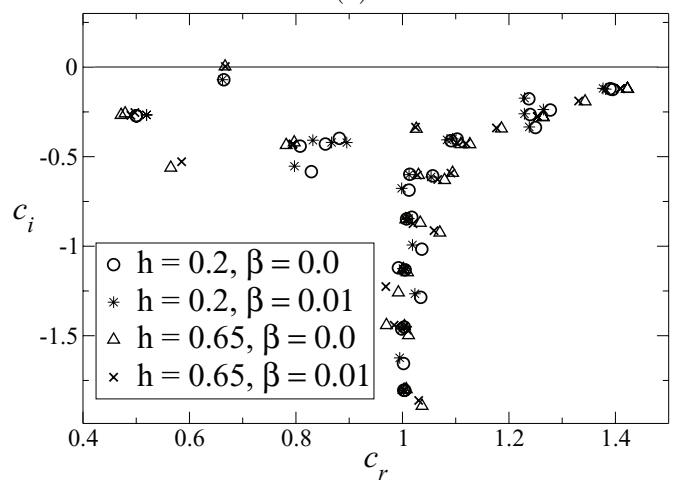

(b)

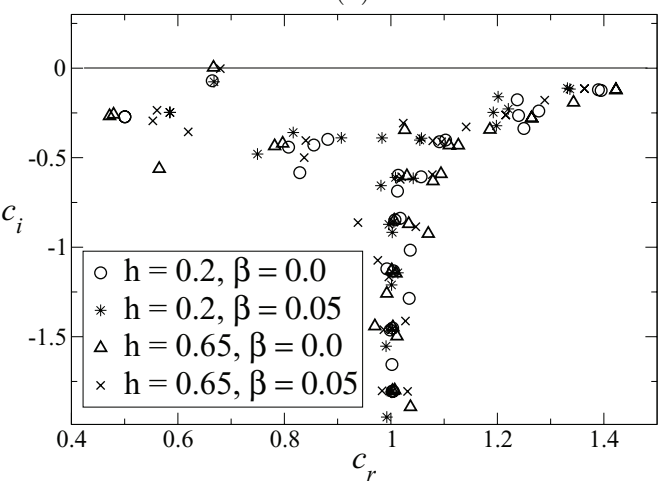

FIG. 5. Eigenspectra for $m=1.2$ for two different values of $h$. The other parameters are $\alpha=1.35, q=0.1, S c=1$, and $R e$ $=400$. (a) $\beta=0.0,0.01$; (b) $\beta=0.0,0.05$. 
(a)

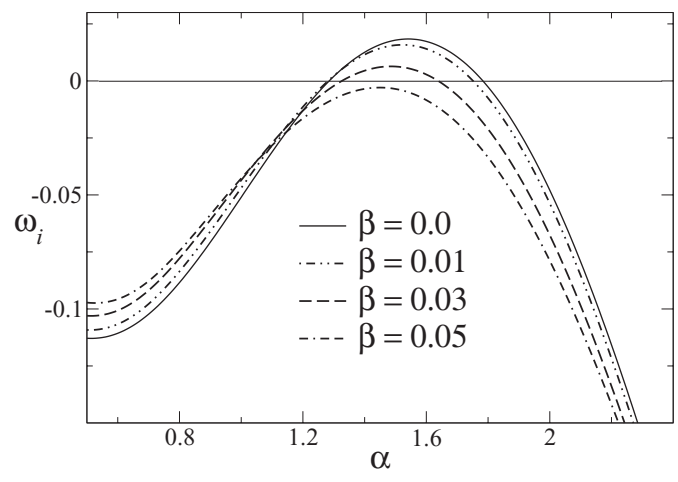

(b)

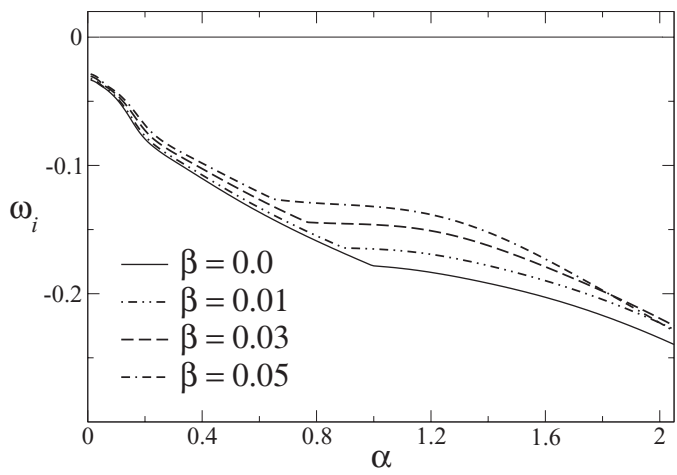

FIG. 6. Effects of slip parameter, $\beta$, on the growth rate $\left(\omega_{i}\right)$ as a function of wave number $(\alpha)$ for $R e=400$ : (a) $m=1.2$ and (b) $m=0.8$. Here, $h=0.65, q=0.1$, and $S c=1$.

slip for viscosity contrast $m=1.2$. This suggests that one can expect interesting instabilities to occur in the case when more viscous fluid is adjacent to the wall $(m=1.2)$ and when the mixed layer and the critical layers overlap. It is of interest to observe what happens when $m<1$ under overlap conditions.

The growth rate $\left(\omega_{i}\right)$ as a function of wave number $\alpha$ is presented in Figs. 6(a) and 6(b) for $m=1.2$ and $m=0.8$, respectively, for $R e=400$. The other parameters are $h=0.65, q=0.1$, and $S c=1$. In Fig. 6(a), there is a range of wave numbers in which $\omega_{i}$ decreases as $\beta$ increases, indicating the stabilizing role of the slip parameter $\beta$. A slip at the wall reduces the shear as can be seen from Figs. 2(e) and 2(f). This suggests that the wall slip stabilizes the flow by decreasing the shear rate. Also note that only a band of wave numbers is unstable; disturbances of shorter wavelengths and small wave numbers are stable. The range of unstable wave numbers decreases with increase in $\beta$. Further, the growth rate for $m=1.2$ is positive for all $\beta \leq 0.03$ whereas this is not the case for $\beta>0.03$. However, in Fig. 6(b) ( $m=0.8)$, the growth rate is negative for all values of the slip parameter $\beta$ considered. For each $\beta$, this behaviour is due to a decrease in wall shear for $m=0.8$ as compared to that for $m=1.2$ (see Figs. 2(a), 2(b), 2(e), and 2(f)). In view of the above, the focus is on the case for $m=1.2$ to understand the instabilities that occur and to study the effects of the parameters on each mode of instability.

The influence of the slip parameter $\beta$ on the stability boundaries for $m=1.2, S c=0.1$, $q=0.1$ is examined in Fig. 7 for different locations of the mixed layer. The configuration corresponds to viscosity increasing towards the wall with slip at $y=1$. For small $h(h=0.2)$, the mixed layer is away from the channel wall and only a TS mode of instability appears at high Reynolds number (Fig. 7(a)) as in the case of a single fluid flow in a channel (Fig. 3(a)) or as in the two-layer miscible fluid flow in a planar rigid channel ${ }^{18}$ (see Fig. 4(a)). An increase in $\beta$ delays the appearance of the TS mode and increases the stability region. It is evident that the critical Reynolds number for the two-fluid miscible layer flow in a channel with wall slip is higher than that in the corresponding miscible two-fluid flow (Fig. 7(a); $\beta=0$ ) and single-fluid flow (Fig. 3(a)) in a rigid channel. Further, in this configuration, the two-fluid miscible channel flow is more stable than the single-fluid flow in a channel with wall slip (from Figs. 7(a) and 3(a) for each $\beta$ ).

As the location of the mixed layer is slightly shifted towards the slippery wall ( $h=0.4$, $m=1.2$ ), a new mode of instability appears at shorter wavelengths ("I" mode; not shown here). With further increase in $h(h=0.63)$, for $m=1.2$ and $q=0.1$, three modes of instabilities, namely, the TS mode, the "I" mode and the "O" mode occupying distinct regions in $\alpha-R e$ plane (Fig. 7(b); $\beta=0.01)$ are observed as in the case of two-fluid rigid channel flow. ${ }^{18}$ At this location of the mixed layer $(h=0.63)$, the inviscid "I" mode becomes dominant for higher $R e$ and shorter wavelength. The overlap "O" mode becomes dominant for relatively smaller $R e$ and wave numbers of order one. The stability boundaries of the "I" mode and the "O" mode are presented for different values of $\beta$ in Figs. 7(c) and 7(d), respectively, for $h=0.63, q=0.1$ and $m=1.2$. It can be seen in Fig. 7(c) 
(a)

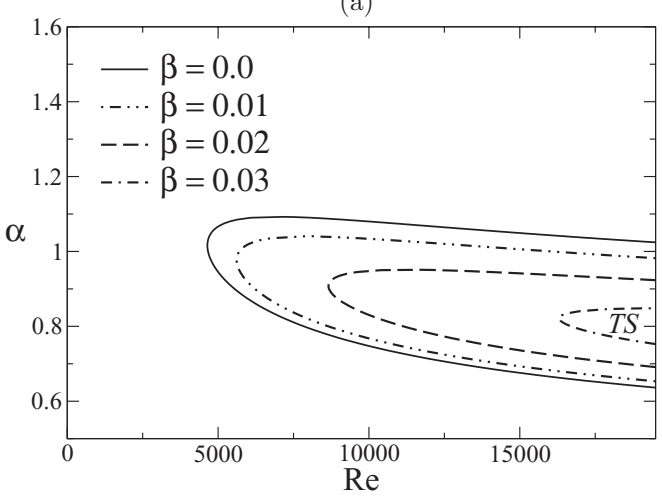

(c)

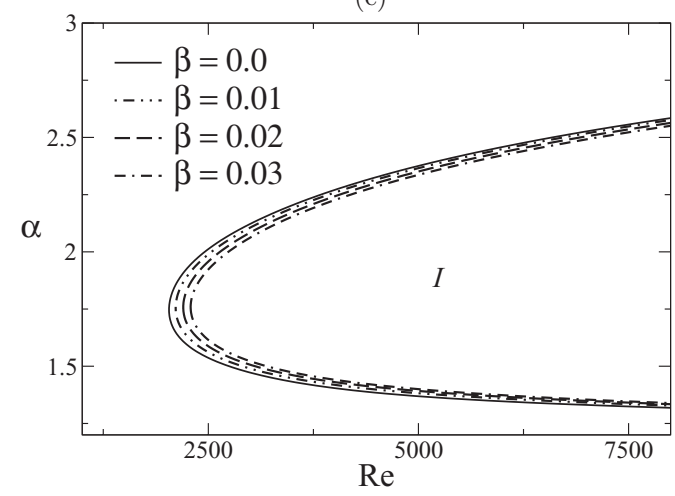

(e)

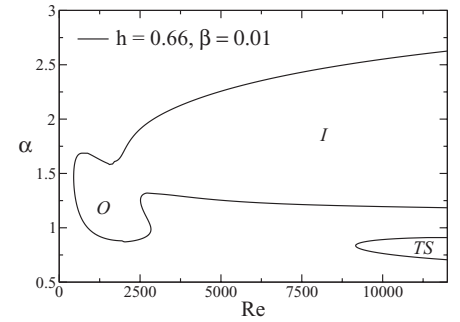

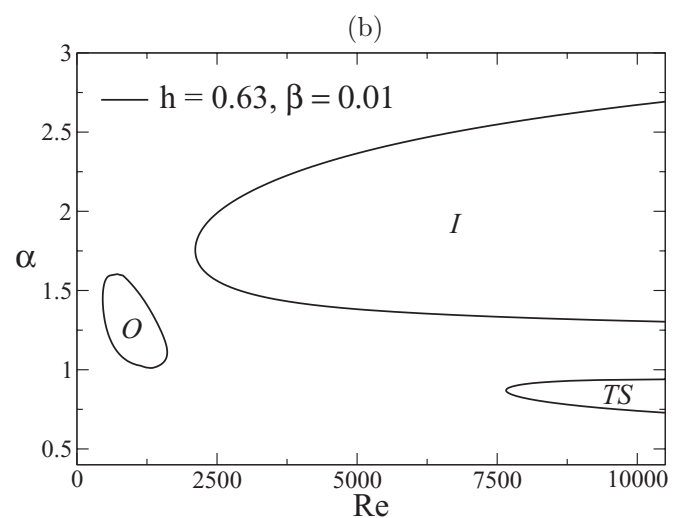

(d)

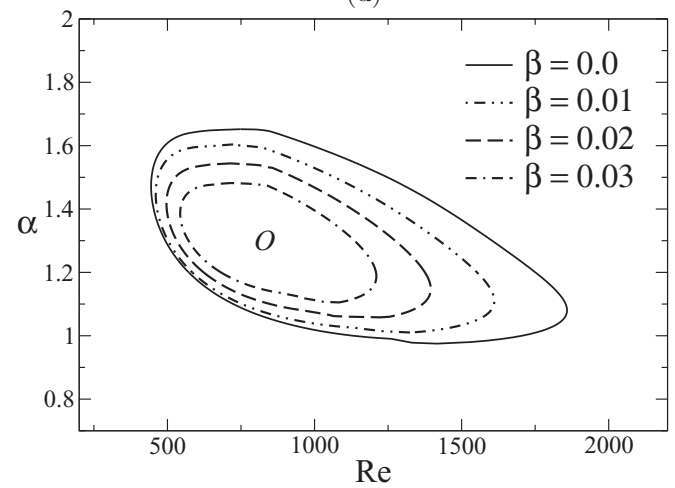

(g)

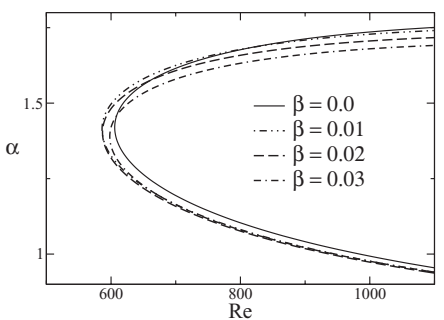

FIG. 7. The neutral stability boundaries for $q=0.1, m=1.2$, and $S c=0.1$. (a) The effects of $\beta$ for $h=0.2$, (b) three distinct modes for $h=0.63 ; \beta=0.01$, (c) effects of $\beta$ on the "I" mode instability for $h=0.63$, (d) the effects of $\beta$ on the "O" mode instability for $h=0.63$, (e) the coalescence of the "I" and the "O" modes for $h=0.66 ; \beta=0.01$, (f) the effects of $\beta$ for $h=$ 0.75 , and (g) zoom of the region $500 \leq R e \leq 1200$ in Fig. 7(f).

that the range of unstable wave numbers decreases with increase in $\beta$ for the "I" mode of instability and it increases the stable region for the "I" mode. Also, as the value of $\beta$ increases, the region of instability for the "O" mode decreases and is limited to relatively smaller Reynolds numbers, but there is no significant change in the critical Reynolds number as $\beta$ increases. The instability occurs at wave numbers of $O(1)$ (Fig. 7(d)) at lower $R e$ for all values of $\beta$ considered and the range of unstable wave numbers decreases with increase in $R e$ for each $\beta$. As $\beta$ increases, the critical Reynolds number increases, indicating that increasing slip delays the onset of "O" mode instability. As $h$ is increased further, the "O" mode and the "I" mode conjoin together (Fig. 7(e)), but the TS mode occupies a distinct region at higher Reynolds number and smaller wave numbers (Fig. 7(e); $\beta$ $=0.01, h=0.66, m=1.2$, and $q=0.1$ ). The stability boundaries for different values of $\beta$ presented in Fig. 7(f) $(h=0.75, q=0.1, m=1.2)$ shows that when the mixed layer is much more closer to the wall, one large region of instability appears due to the coalescence of the three modes of instability. It is noted that, a small increase in $\beta$ destabilizes the flow system, which is stabilized 
(a)

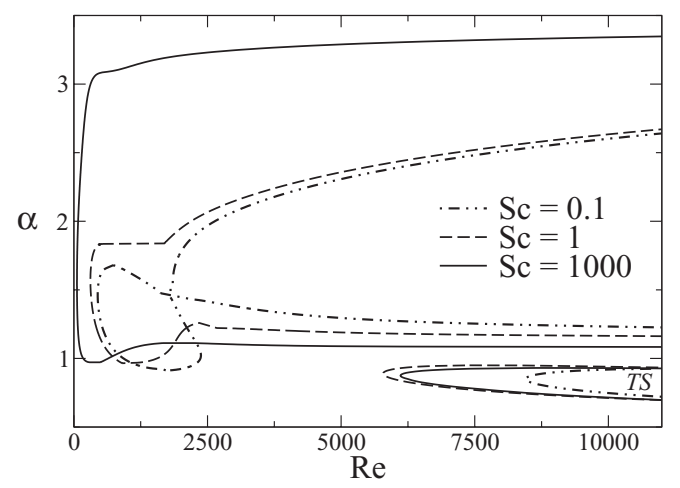

(b)

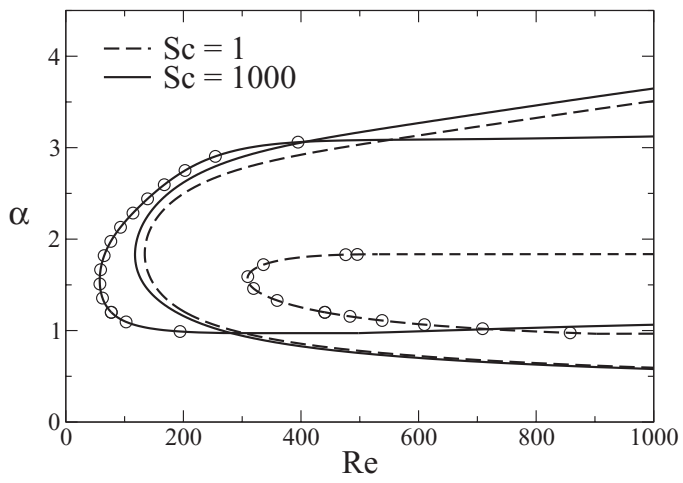

FIG. 8. The effect of $S c$ on the neutral stability, for $h=0.65, q=0.1$, and $\beta=0.01$ with (a) $m=1.2$; (b) $m=1.2$ (with symbols) and $m=1.8$ (without symbols).

with further increase in $\beta$, as it is evident from Fig. 7(g) (the zoom of the region for $500 \leq R e \leq$ 1200 in Fig. 7(f)). This indicates a non-monotonic behaviour with respect to $\beta$. Further, the range of unstable wave numbers decreases with an increase in $\beta$, for higher Reynolds numbers. Also, for large Reynolds numbers, shorter wavelength disturbances are stabilized by slip at the wall, whereas the longer wavelength disturbances are not affected significantly by the slip at the wall. The results reveal that, if required, depending upon relevant applications, it is possible to either destabilize or stabilize miscible two-fluid flow system in a channel with wall slip for the above configuration using appropriate slip length as compared to the same flow system in a rigid channel.

Apart from Fig. 4 presented for $S c=0$, for the sake of validating the developed code, other results in this study are presented for valid and realistic values of $S c$ well within the regime (Appendix A). As pointed out by Govindarajan ${ }^{18}$ and Usha et al. ${ }^{52}$ non-parallel flow effects have to be examined, even at high Reynolds number, for the case when the two fluids diffuse into each other very rapidly. It is of interest to understand the effects of diffusion on the instabilities that occur for the configuration when a highly viscous fluid is closer to the wall under overlap conditions. Fig. 8(a) presents the results for $h=0.65, q=0.1, m=1.2$ when $\beta=0.01$ for different values of $S c$. For $S c=0.1$ the stability boundaries of the "I" and the "O" mode contain a bifurcation point. A small change in $S c$ (from $S c=0.1$ to $S c=1$ ) has changed the scenario in the result for $S c=0.1$. The O-mode and the inviscid mode (I-mode) conjoin together while the TS mode occurs distinctly. The effect of increasing $S c$ is to destabilize the flow system in which both the long and short wavelength disturbances are influenced, for large Reynolds numbers. At an intermediate level of diffusivity ( $S c$ $=1000$ ), the flow is unstable at very low Reynolds numbers and over a wide range of wave numbers. Here again, there is coalescence of the "O" and "I" modes enclosing a large unstable region in the $\alpha-R e$ plane. The TS mode occurs distinctly and is stabilized at this intermediate level of diffusivity.

Fig. 8(b) shows the diffusion effects for a configuration with higher viscosity ratio $(m=1.8)$. An increase in $S c$ destabilizes the flow system for both $m=1.2$ and $m=1.8$. However, diffusion effects are more significant for $m=1.2$ than for $m=1.8$. In view of this, the results are presented for values of $m$ close to $m=1$.

Fig. 7(f) presents some curious features with regard to slip parameter $\beta$, when $S c=0.1$. It is of interest to see what happens with an increase in Sc. This is analyzed in Figs. 9(a) and 9(b). Fig. 9(a) shows that at $R e=1200$, the growth rate is positive for a range of wave numbers and it decreases with increase in $\beta$. The same trend is observed for $R e=1200$ when $S c=10$ in Fig. 9(b). At $R e=700$, the slip exhibits a non-monotonic behaviour (Figs. 9(a) and 9(b)); and with increase in $S c$, the stabilizing effect of the slip parameter occurs at a higher value of $\beta$. Also, as $S c$ increases, the growth rate is much more than that for $S c=0.1$ and the range of unstable wave numbers is increased. This indicates the destabilizing effect of the diffusivity parameter $S c$.

Next, the neutral stability boundaries for $S c=1000, m=1.2$, and $q=0.1$ are presented in Fig. 10(a) (for $h=0.2$ ) and Fig. 10(b) (for $h=0.7$ ). The TS mode of instability is the only unstable 
(a)

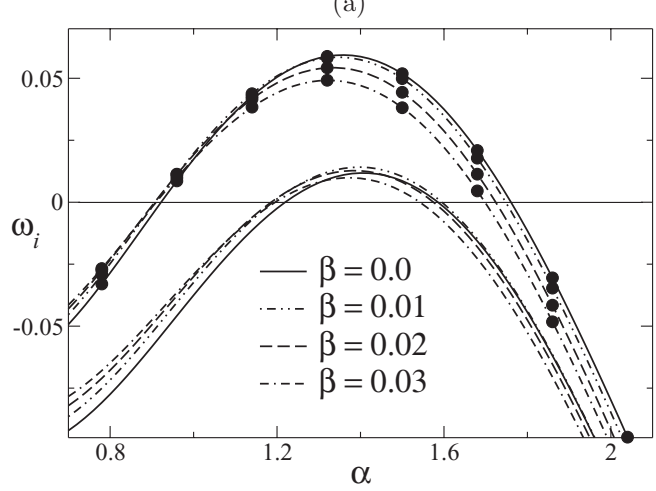

(b)

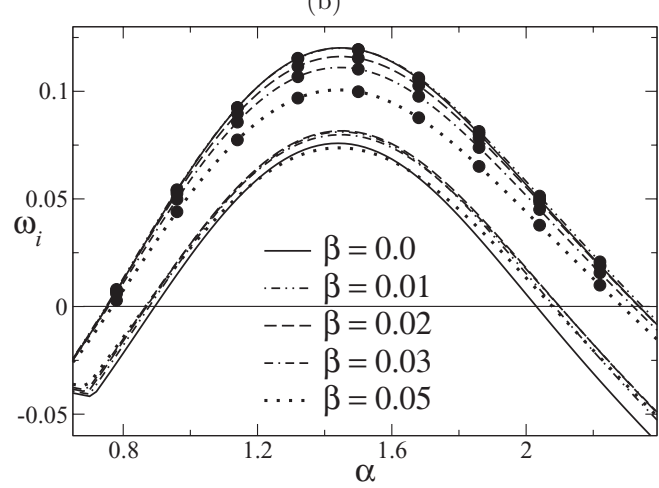

FIG. 9. Growth rate curves for $h=0.75, q=0.1, m=1.2, R e=700$ (without symbols), and $R e=1200$ (with symbols): (a) $S c=0.1$; (b) $S c=10$.

mode for the case $h=0.2$ and the critical Reynolds number for each $\beta$ for $S c=1000$ is slightly smaller than that for $S c=0.1$ (see Fig. 7(a)). This indicates the destabilizing role of $S c$ for $h=0.2$. As $h$ is increased to $h=0.7$ (see Fig. 10(b)) the diffusivity parameter exhibits a stronger influence on destabilizing the flow. In this case, the flow becomes unstable for smaller Reynolds number and higher wave numbers as compared to the corresponding results for $S c=0.1$ (not shown here). For $S c=1000$ (Fig. 10(b)), the coalescence of the "O," "I" modes and the TS mode instability exist in distinct regions of $\alpha-R e$ plane. Fig. 10(c) presents a zoom of the region $0 \leq R e \leq 400$ in Fig. 10(b).

(a)

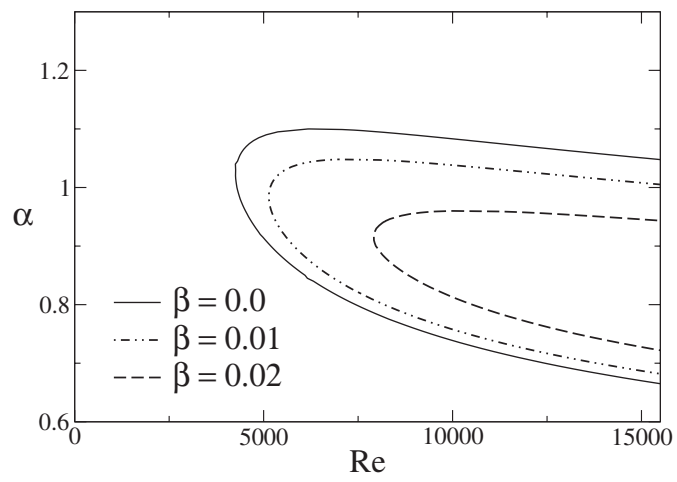

(b)

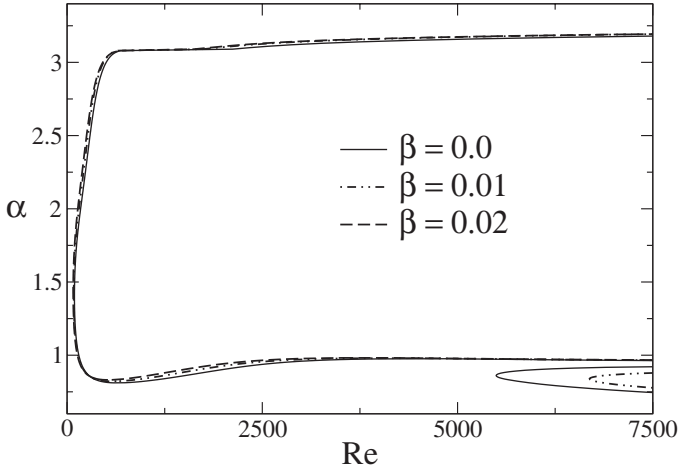

(c)

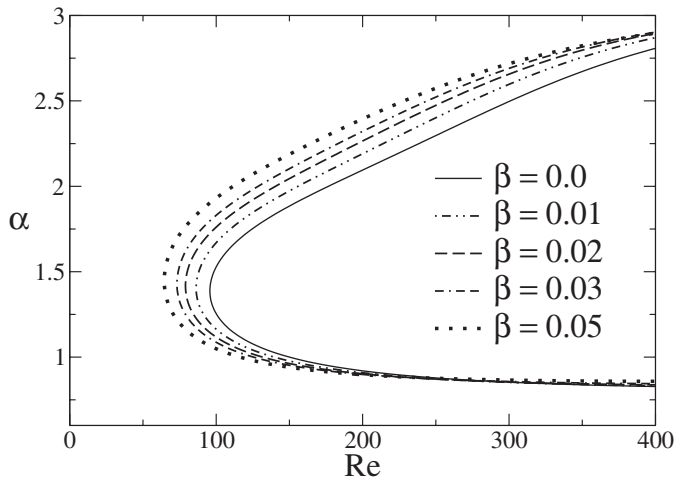

FIG. 10. The neutral stability boundaries for different values of $\beta$ with $S c=1000, m=1.2$, and $q=0.1$; (a) $h=0.2$; (b) $h$ $=0.7$, and (c) zoom of the region $0 \leq R e \leq 400$ in Fig. 10(b). 
(a)

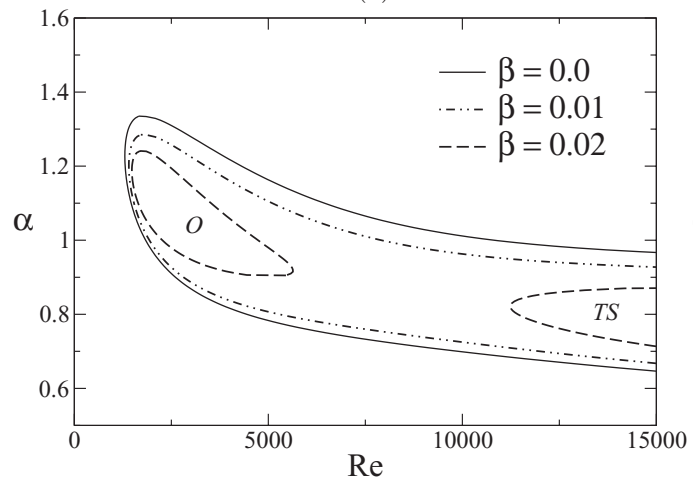

(c)

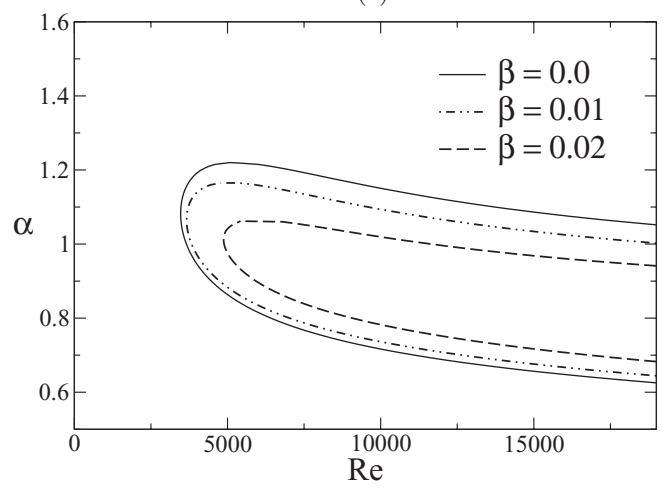

(b)

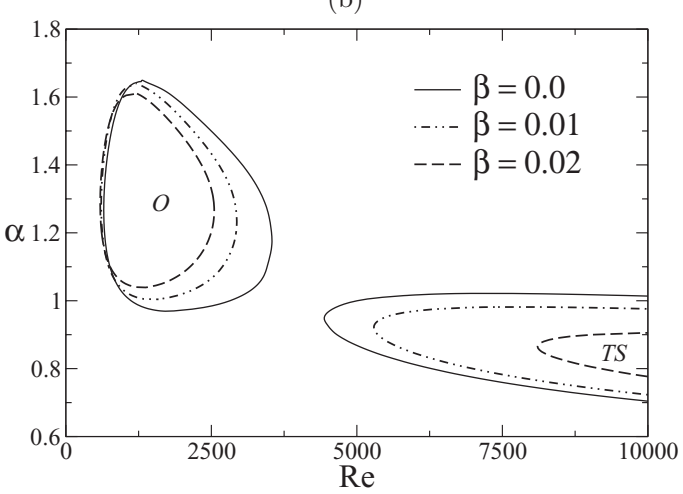

(d)

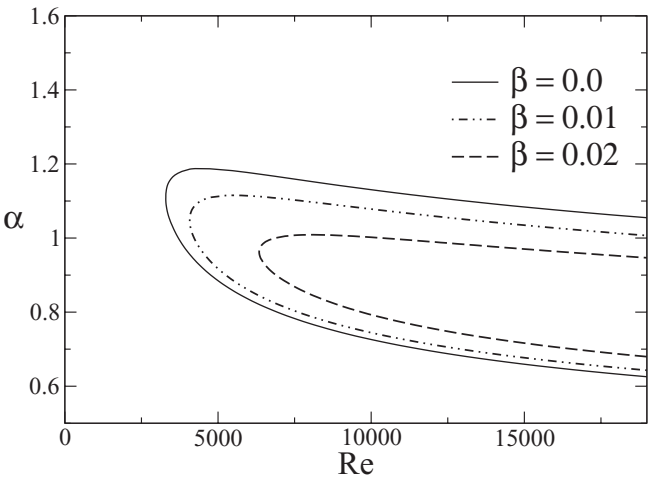

FIG. 11. Effects of $\beta$ on the neutral stability boundaries for (a) $S c=1$ and (b) $S c=1000$, for $m=1.05$ and $h=0.75$; (c) $S c$ $=1$ and (d) $S c=1000$, for $m=0.95$ and $h=0.75$. In all the panels, the value of $q$ is 0.1 .

The critical Reynolds number decreases with increase in $\beta$, indicating the destabilizing role of $\beta$ at this value of $S c$.

Now, the effects of diffusivity are examined for two different viscosity contrasts ( $m=1.05$ and $m=0.95$ ). Fig. 11(a) shows the results for different $\beta$ when the fluid adjacent to the wall is highly viscous ( $m=1.05$ ) for $h=0.75, q=0.1$, and $S c=1$. The TS and the "O" modes are conjoined and a large region of instability appears for moderate to large Reynolds number for $\beta=0.0$ and 0.01 . However, the "O" mode and TS mode occupy distinct regions in the $\alpha-R e$ plane for $\beta=0.02$. It is also observed that the "I" mode does not exist for this set of parameters. This is in striking contrast with the results presented in Fig. 8 for $S c=1, h=0.65, \beta=0.01$, where the "I" mode exists and conjoins with the "O" mode and the TS mode occupies a distinct region. The critical Reynolds number $\left(R e_{c r}\right)$ for the onset of instability increases with an increase in wall slip, characterized by $\beta$. The critical Reynolds number $\left(R e_{c r}\right)$ for miscible two-fluid channel flow with no-slip/slip at the wall is much lower than that for a single fluid flow in a channel with no-slip/slip at the wall (see Fig. 3(a)). This indicates the destabilizing role of viscosity stratified layer when it is located closer to the channel wall. As $S c$ increases ( $S c=1000$; intermediate level of diffusivity), it can be seen in Fig. 11(b) that the "O" mode instability occurs and for each value of $\beta$, it appears in a domain distinct from that of the TS mode. The unstable region of the "O" mode instability decreases with increase in the value of $\beta$. At this $S c$, the "O" mode is the dominant mode of instability. This instability occurs for higher wave numbers for $S c=1000$ than for $S c=1$, for each $\beta$. The Reynolds number at which instabilities arise for $S c=1000$ is much smaller than the corresponding Reynolds number for $S c=1$. This indicates the destabilizing role of the diffusivity parameter $(S c)$ for this configuration $(m=1.05$ and $h=0.75$ ). The critical Reynolds number decreases with increase in $\beta$ for the values of $\beta$ shown in figure, indicating the destabilizing effect of $\beta$. 


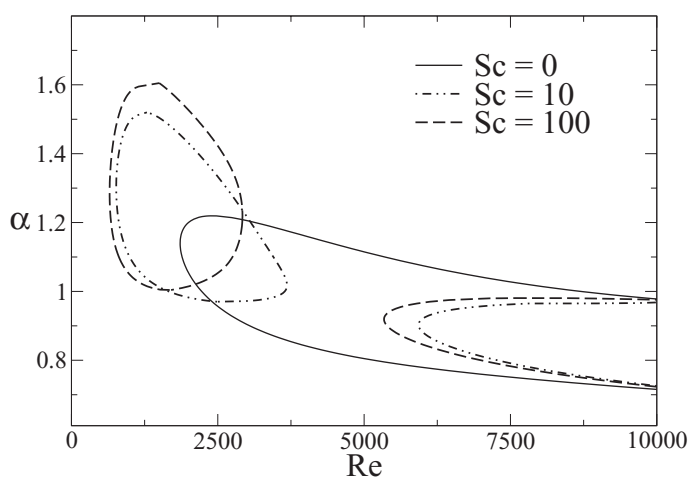

FIG. 12. The effect of $S c$ on the neutral stability boundaries, for $h=0.75, q=0.1, m=1.05$, and $\beta=0.01$.

The corresponding results for the case when a fluid with lower viscosity is adjacent to the wall ( $m=0.95$ ) are presented in Figs. 11(c) and 11(d) for $S c=1$ and $S c=1000$, respectively, for $h$ $=0.75$ and $q=0.1$. The results show that the TS mode is the dominating mode of instability and the flow is stabilized as $S c$ increases from 1 (Fig. 11(c)) to 1000 (Fig. 11(d)). It is apparent that a non-zero value of $\beta$ is stabilizing the flow dynamics for both the $S c$ values considered.

The "O" mode and TS mode occupy distinct regions in the $\alpha-R e$ plane for $S c=1$ (Fig. 11(a)) when $\beta \geq 0.02$ while this happens for $\beta \geq 0.0$ when $S c=1000$ (Fig. 11(b)). The characteristics of these modes for different values of $S c$ between $S c=1$ and $S c=1000$ when $m=1.05$, $h=0.75$ are displayed in Fig. 12. The result for $S c=0$ is also incorporated and in this case, the two modes are conjoined to form a single region. At high diffusivity level $(S c=10)$, the TS and overlap modes occupy distinct regions for $\beta=0.01$ which is in contrast to that for $S c=1, \beta=0.01$ (see Fig. 11(a)). At a level of diffusivity corresponding to $S c=100$, the same trend as above is observed. The critical Reynolds number decreases indicating the destabilizing effect of $S c$ and the unstable region for "O" mode extends to higher wave numbers and shrinks to small Reynolds numbers for $S c$ $=100$ than for $S c=10$.

Fig. 13 presents the influence of diffusivity on the critical Reynolds number for the viscosity ratio $m=1.2$ for $h=0.7, q=0.1$. The flow is unstable in the region above a given curve. The critical Reynolds number decreases with increase in $S c$ beyond $S c=0.1$, indicating the destabilizing effect of the decreasing diffusivity for $m=1.2$. For this viscosity contrast, there is a non-monotonic behaviour with respect to slip parameter $\beta$ up to $S c=0.1$; the flow is destabilized in a channel with velocity slip beyond $S c=0.1$ as compared to that in a rigid channel.

The computations presented so far show that small changes in the viscosity ratio $(m)$ close to 1 significantly affect the stability properties of the fluid. The effects of viscosity contrast on the

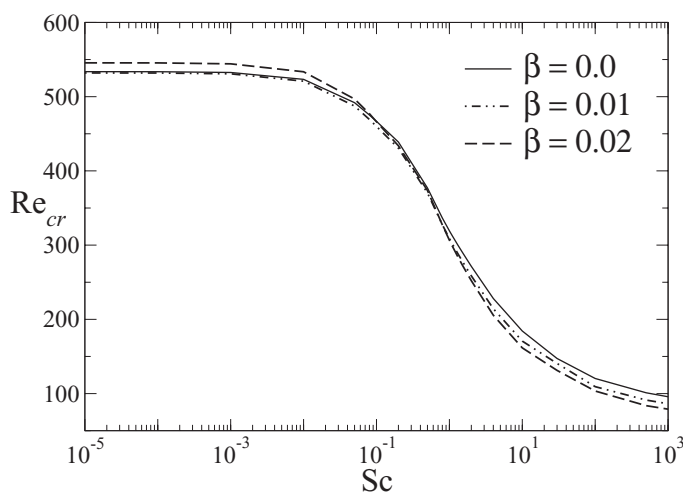

FIG. 13. The critical Reynolds number as a function of $S c$ for $h=0.7, q=0.1$, and $m=1.2$. 
(a)

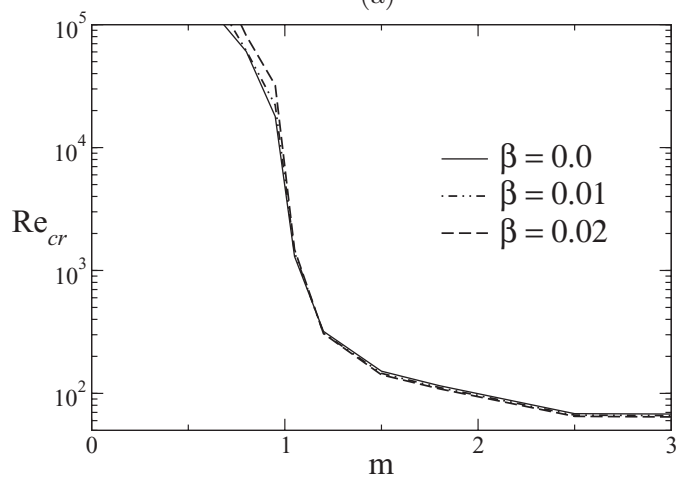

(c)

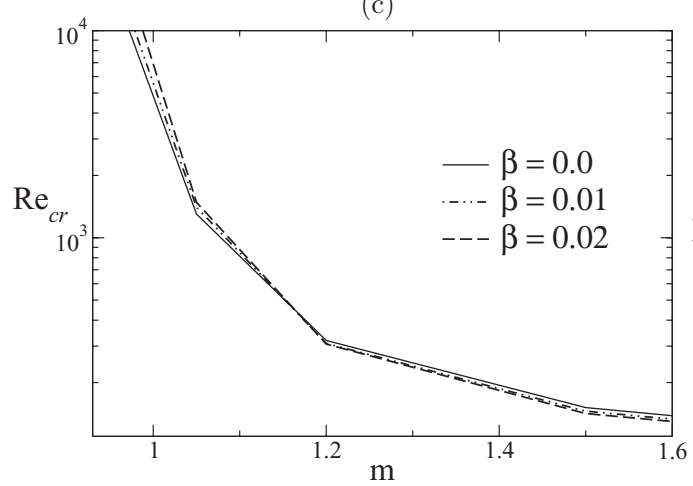

(b)

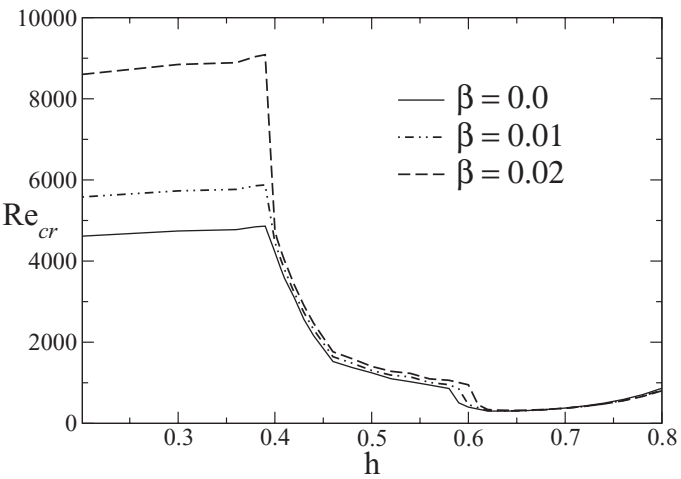

(d)

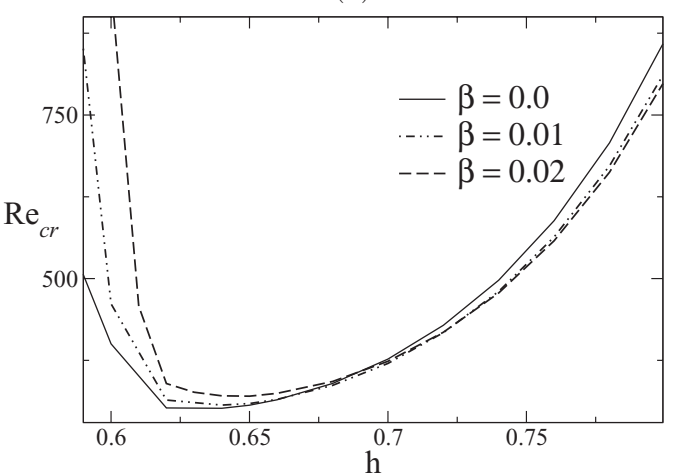

FIG. 14. (a) The critical Reynolds number as a function of $m$. The computations are performed with $(h, m)=(0.6,>1.8)$, $(0.65,1.8),(0.7,1.2),(0.75,1.05),(0.85,0.95),(0.87,0.8)$, and $(0.89,0.6)$. (b) The critical Reynolds number as a function of $h$ and $m=1.2$. (c) Zoomed portion of Fig. 14(a) close to $m=1$. (d) Zoomed region of Fig. 14(b) for $0.55 \leq h \leq 0.8$. In all the panels $q=0.1$ and $S c=1$.

critical Reynolds number presented in Fig. 14(a) also confirm this result. The velocity slip at the wall stabilizes the flow slightly for $m \leq 1.15$ and a reverse trend is displayed for $m \geq 1.15$ (highly viscous fluid is close to the channel wall), under overlap conditions of the critical and the mixed layers (see Fig. 14(c); zoom of the region close to $m=1$ in Fig. 14(a)). In Fig. 14(b), the effects of location of the mixed layer on the critical Reynolds number are examined for different values of $\beta$ with $m=1.2$. There is a drastic decrease in the value of $R e_{c r}$ around $h=0.4$ up to which the TS mode is the dominant mode. Beyond $h=0.4$ to 0.6 the inviscid mode ("I" mode) dominates. The critical layer falls in the region of the viscosity stratified layer for $h>0.6$. This causes the emergence of the overlap mode as discussed above, which is the most unstable mode. Inspection of this plot also reveals the destabilizing effect of the slip at the channel wall for the "O" mode instability (see Fig. 14(d); zoomed portion for $0.55 \leq h \leq 0.8$ in Fig. 14(b)). On the other hand, the flow is stabilized by the velocity slip at the wall for the TS and the inviscid modes.

\section{CONCLUSIONS}

The present study examines the effects of wall slip on the instabilities in plane Poiseuille flow of two miscible layers of fluids of same density but different viscosities, in a channel with velocity slip at the wall. The wall slip has been shown to have significant effect on the stability of the flow system. The slippery wall plays a dual role of either stabilizing or destabilizing the flow system as compared to that in a rigid channel.

An overlap mode has been shown to appear when the critical layer of the dominant disturbance overlaps the viscosity stratified layer, for high mass diffusivity of the two fluids, in the absence of velocity slip at the walls by Govindarajan. ${ }^{18}$ Such a mode also appears in the present case for 
moderate wave numbers and small Reynolds numbers. Figs. 5(a) and 5(b) in which eigenvalue spectra are presented for $R e=400$ when the mixed layer is located at $h=0.2$ and $h=0.65$ clearly indicate that the overlap instability occurs due to destabilization of an existing mode. This may be attributed to the increase in the disturbance kinetic energy due to the effect of overlap of viscosity stratified layer with the critical layer. The spectra in the two cases (Fig. 5) appear similar but the flow is unstable when the mixed layer and the critical layer of dominant instability overlap with each other (for $h=0.65$ ), whereas for $h=0.2$ all the modes are stable. It can also be seen that the unstable mode for $h=0.65$ is not a new eigenmode (like in case of pure interfacial flows), but a stable mode (for $h=0.2$ ) becoming unstable due to the overlap of viscosity stratified layer with the critical layer. The unstable region for "O" mode decreases with increase in the value of $\beta$, but the critical Reynolds number is not affected much by the slip parameter $\beta$.

The dimensionless Schmidt number characterizing the diffusivity affects the overlap mode significantly and at any fixed value of slip parameter, $\beta$, the degree of destabilization increases with increase in Schmidt number $(S c)$. There is stabilization of the flow system for the slip parameter $\beta$ $\geq 0.02$ when $S c \leq 0.1$ and destabilization of the flow system as the slip at the wall increases when $S c>0.1$ (see Fig. 13).

The effects of slip on the TS mode and the inviscid mode which exist along with the overlap mode under certain conditions on the location of the viscosity stratified layer have also been examined as diffusivity, the ratio of viscosities, and the location of the viscosity-stratified layer are varied.

The two-fluid miscible flow in a slippery channel is more stable than either the corresponding two-fluid miscible flow in a rigid channel or a single fluid flow in a rigid/slippery channel (see Figs. 3(a) and 7(a)), when a higher viscous fluid is adjacent to the wall and the mixed layer and the critical layer are well separated. With the same viscosity contrast when the mixed layer and the critical layer overlap, the miscible two-fluid channel flow with slip/no-slip at the wall is more unstable than the corresponding single fluid flow in a channel with slip/no-slip (see Figs. 3(a) and 11(a)). Further, the stability characteristics of the miscible two-fluid channel flows with slip are different from both the limiting cases of viscosity-stratified flows with sharp jump and continuously stratified flows in a channel with slip. The overlap mode instability is absent in both the limiting cases. In the present study, a configuration with less viscous fluid closer to the wall is more stable than that with highly viscous fluid adjacent to the wall. On the other hand, the critical Reynolds number increases with increase in viscosity ratio for the interface dominated flow in a channel with slip ${ }^{46}$ indicating a reverse trend as compared to that in the present study.

Although the present results are analogous to those in Govindarajan for a rigid channel, the message from the present study is that the flow system considered by Govindarajan ${ }^{18}$ can be further stabilized or destabilized if one imposes velocity slip at the channel wall. It is well known that creating a small viscosity stratification in the fluid is one of the effective controlling strategies for delaying the occurrence of fluid flow turbulence. It has been shown by several investigators that a laminar wall-bounded shear flow consisting of two layers of fluids of different viscosities is significantly stabilized whenever the fluid with less viscosity is adjacent to the wall, provided that the viscous interface is located near the critical layer. The present study shows that the stabilizing or destabilizing effect can be further enhanced by taking the channel with velocity slip at the wall.

The theoretical studies by $\mathrm{Kim}$ and $\mathrm{Kim},{ }^{30} \mathrm{Gan}$ and $\mathrm{Wu},{ }^{37}$ and the experimental investigations ${ }^{28,29}$ show that flow over a hydrophobic surface can be analyzed by the Navier-Stokes equations with slip boundary condition. We also infer from Beskok and Karniadkis ${ }^{41}$ that if the slip conditions are used, the Navier-Stokes equations are valid for slip length up to 0.1. This suggests that the results of the present study may be used for understanding the stability of miscible two-fluid flow in a channel with hydrophobic surface which can be modeled as surfaces with velocity slip at the wall. The present study also could be useful in micro-electromechanical systems and flow in microfluidic channels, where there is an increasing evidence that the boundary condition of slip type is needed rather than no-slip boundary condition at the walls to model the flow dynamics accurately.

In view of the relevance of the nonmodal stability analysis than the modal stability analysis in the subcritical transition in channel flows, it is of interest to quantify the effect of wall slip on the nonmodal analysis for the present flow system and this will be explored in our future study. 
The present study also opens the possibility of accurate experimental set up and direct numerical simulation.

\section{ACKNOWLEDGMENTS}

The authors thank the referees for their very useful, valuable comments and suggestions. The discussions with Professor Rama Govindarajan have been very inspiring and the authors thank her for this. The authors also thank the Editor, Professor L. Gary Leal for his encouraging and supporting remarks and suggestions.

\section{APPENDIX A: VALIDITY OF PARALLEL FLOW ASSUMPTION}

The present study is based on the parallel flow assumption in the mixed layer. This is equivalent to considering that the variations of the gradients in flow variables at the steady state and the thickness $q$ of the mixed region have a much larger length scale than the disturbance wavelength. The following discussion shows that the above assumption is justified for slow diffusion of the fluids (higher values of Péclet numbers).

Let a splitter plate be located at $x<x_{0}$, at a constant $y$ and let the parallel streams of two miscible fluids flow on both sides of this plate. The streams come into contact with each other at $x=x_{0}$. The two fluids begin to mix with each other for $x>x_{0}$, thus producing a stratified layer. The thickness " $q$ " of this layer grows as the fluids move downstream and therefore $q$ is a function of $x$. In what follows, it is shown that the thickness of the mixed layer varies slowly in $x$, i.e., $\partial q / \partial x \ll 1$.

At any location, the steady mean concentration satisfies the equation

$$
U \frac{\partial s}{\partial x}+V \frac{\partial s}{\partial y}=\frac{1}{P e}\left[\frac{\partial^{2} s}{\partial x^{2}}+\frac{\partial^{2} s}{\partial y^{2}}\right] .
$$

Under the boundary layer approximation, $V \ll U$ and $\frac{\partial^{2}}{\partial x^{2}} \ll \frac{\partial^{2}}{\partial y^{2}}$ and this yields

$$
U \frac{\partial s}{\partial x} \simeq \frac{1}{P e} \frac{\partial^{2} s}{\partial y^{2}}
$$

Also using the same approximation, we know that $U \sim O(1), y \sim \sqrt{v}$, where $v$ is the viscosity. Therefore, $q s \sim O\left(y^{2}\right)$ since $s$ is the mean concentration over the mixed layer of thickness $q$. This implies that $\partial s / \partial x \simeq \frac{1}{q} O(1 / P e)$ (from Eq. (A2)). So, for large values of $P e, \partial s / \partial x$ is very small, showing that the downstream variation of $s$ is very small which in turn implies that the changes in the thickness $q$ of the mixed layer along the $x$-direction is very small.

Alternatively, if we assume a similar solution $s(y / q(x)) \simeq s(\xi)($ where $\xi=(y / q(x)))$ for Eq. (A2), we will get

$$
U \frac{d s}{d \xi}\left(-\frac{\xi}{q} \frac{d q}{d x}\right) \simeq \frac{1}{P e}\left(\frac{d^{2} s}{d \xi^{2}} \frac{1}{q^{2}}\right)
$$

As a consequence,

$$
\frac{1}{q} \frac{d q}{d x} \sim \frac{1}{q^{2} P e} \Rightarrow \frac{d q}{d x} \sim \frac{1}{q} O(P e)^{-1}
$$

Thus, the downstream growth of mixed layer is inversely proportional to the Péclet number as $U$ and $\xi$ are of $O(1)$ and $O\left(\frac{d s}{d \xi}\right) \simeq O\left(\frac{d^{2} s}{d \xi^{2}}\right)$, which confirms that for the Reynolds and Schmidt numbers considered in the present study, the assumption of uniform thickness of viscosity stratified layer is justified. 


\section{APPENDIX B: RELATION TO THE INTERFACIAL PERTURBATION IN THE IMMISCIBLE LIMIT}

The whole viscosity field is perturbed by introducing a viscosity perturbation $\mu$ (expressed in terms of "s") to the base state viscosity. In view of this, there is no need to separately perturb the interface variable $h$ between the fluid $i(i=1,2)$ and the mixed layer.

In what follows, we show that, in the absence of diffusion (i.e., $P e \rightarrow \infty$ ), the viscosity along a particle path (the perturbed interface) is constant. Consider the linearized equation for the perturbation in viscosity (Eq. (19)). The condition that the viscosity along a particle path changes only by viscosity diffusion yields the equation

$$
\frac{D}{D t}\left(\mu_{B}+\mu\right)=\frac{1}{P e} \nabla^{2}\left(\mu_{B}+\mu\right)
$$

where $\frac{D}{D t} \equiv \frac{\partial}{\partial t}+\left(U_{B}+u\right) \frac{\partial}{\partial x}+(v) \frac{\partial}{\partial y}$. In the absence of diffusion $(P e \rightarrow \infty)$, the equation gives

$$
\frac{D}{D t}\left(\mu_{B}+\mu\right)=0
$$

which shows that the lines of constant viscosity are the same as the particle paths. By definition, the perturbed interface between fluid $i(i=1,2)$ and the mixed layer is a line of constant viscosity and therefore follows a particle path (Eq. (B1)).

This result is the same as that in the case of immiscible configurations, as in immiscible systems the equation for the interface perturbation $h$ states that the viscosity along a particle path, namely, the perturbed interface, is constant. This clearly establishes the relation to the interfacial disturbance in the immiscible limit.

${ }^{1}$ R. Govindarajan and K. C. Sahu, "Instabilities in viscosity-stratified flows," Annu. Rev. Fluid Mech. 46, 331-353 (2014).

${ }^{2}$ C. S. Yih, "Instability due to viscous stratification," J. Fluid Mech. 27, 337 (1967).

${ }^{3}$ A. P. Hooper and W. G. C. Boyd, "Shear flow instability at the interface between two fluids," J. Fluid Mech. 128, 507 (1983).

${ }^{4}$ E. J. Hinch, "A note on the mechanism of the instability at the interface between two shearing fluids," J. Fluid Mech. 144, 463 (1984).

${ }^{5}$ Y. Renardy, "Viscosity and density stratification in vertical Poiseuille flow," Phys. Fluids 30, 1638 (1987).

${ }^{6}$ D. D. Joseph and Y. Y. Renardy, Fundamentals of Two-Fluid Dynamics. Part II. Lubricated Transport, Drops and Miscible Fluids, Interdisciplinary Applied Mathematics Vol. 4 (Springer, New York, 1992).

${ }^{7}$ M. J. South and A. P. Hooper, "Linear growth in two-fluid plane Poiseuille flow," J. Fluid Mech. 381, 121 (1999).

${ }^{8}$ S. G. Yiantsios and B. G. Higgins, "Linear stability of plane Poiseuille flow of two superposed fluids," Phys. Fluids 31, 3225 (1988).

${ }^{9}$ S. G. Yiantsios and B. G. Higgins, "Erratum: Linear stability of plane Poiseuille flow of two superposed fluids [Phys. Fluids 31, 3225 (1988)]," Phys. Fluids A 1(5), 897 (1989).

${ }^{10}$ A. Pinarbasi and A. Liakopoulos, "Stability of two-layer Poiseuille flow of Carreau-Yasuda and Bingham-like fluids," J. Non-Newtonian Fluid Mech. 57, 227 (1995).

${ }^{11}$ S. N. Timoshin and A. P. Hooper, "Mode coalescence in a two-fluid boundary layer stability problem," Phys. Fluids 12, 1969 (2000)

${ }^{12}$ P. Laure, H. Le. Meur, Y. Demay, J. C. Saut, and S. Scotto, "Linear stability of multilayer plane Poiseuille flows of Oldroyd B fluids," J. Non-Newtonian Fluid Mech. 71, 1 (1997).

${ }^{13}$ A. R. Wazzan, T. T. Okamura, and A. M. O. Smith, "The stability of water flow over heated and cooled flat plates," Trans. ASME C: Heat Trans. 90, 109 (1968).

${ }^{14}$ D. P. Wall and S. K. Wilson, "The linear stability of channel flow of fluid with temperature dependent viscosity," J. Fluid Mech. 323, 107 (1996).

${ }^{15}$ D. P. Wall and S. K. Wilson, "The linear stability of flat-plate boundary layer flow of fluid with temperature dependent viscosity," Phys. Fluids 9, 2885 (1997).

${ }^{16}$ B. T. Ranganathan and R. Govindarajan, "Stabilisation and destabilisation of channel flow by location of viscosity-stratified fluid layer," Phys. Fluids 13(1), 1 (2001).

${ }^{17}$ P. Ern, F. Charru, and P. Luchini, "Stability analysis of a shear flow with strongly stratified viscosity," J. Fluid Mech. 496, 295 (2003).

${ }^{18}$ R. Govindarajan, "Effect of miscibility on the linear instability of two-fluid channel flow," Int. J. Multiphase Flow 30, 1177 (2004).

${ }^{19}$ K. C. Sahu, H. Ding, P. Valluri, and O. K. Matar, "Linear stability analysis and numerical simulation of miscible channel flows," Phys. Fluids 21, 042104 (2009).

${ }^{20}$ L. Talon and E. Meiburg, "Plane Poiseuille flow of miscible layers with different viscosities: instabilities in the Stokes flow regime," J. Fluid Mech. 686, 484 (2011).

${ }^{21}$ Y. Zhu and S. Granick, "Rate-dependent slip of Newtonian liquid at smooth surfaces," Phys. Rev. Lett. 87, 096105 (2001). 
${ }^{22}$ P. A. Thompson and S. M. Troian, "A general boundary condition for liquid flow at solid surfaces," Nature 389, 360 (1997).

${ }^{23}$ J. W. Hoyt, "Hydrodynamic drag reduction due to fish slimes," Swimming and Flying in Nature (Springer, 1975), Vol. 2, p. 653.

${ }^{24}$ D. W. Bechert, M. Bruse, W. Hage, and R. Meyer, "Fluid mechanics of biological surfaces and their technological applications," Naturwissenschaften 87, 157 (2000).

${ }^{25}$ E. H. Kennard, Kinetic Theory of Gases (McGraw-Hill, New York, 1938).

${ }^{26}$ G. A. Bird, Molecular Gas Dynamics and the Direct Simulation of Gas Flows (Oxford University Press, Great Clarendon Street, Oxford, 1994).

${ }^{27}$ M. M. Denn, "Extrusion instabilities and wall slip,” Annu. Rev. Fluid Mech. 33, 265 (2001).

${ }^{28}$ D. C. Tretheway and C. D. Meinhart, "Apparent fluid slip at hydrophobic microchannel walls," Phys. Fluids 14, L9 (2002)

${ }^{29}$ C. H. Choi, K. J. A. Westin, and K. S. Breur, "Apparent slip flows in hydrophilic and hydrophobic microchannels," Phys. Fluids 15, 2897 (2003).

${ }^{30}$ J. Kim and C. J. Kim, "Nanostructured surfaces for dramatic reduction of flow resistance in droplet-based microfluidics," in Technical Digest, IEEE Conference on MEMS, Las Vegas, NV (IEEE, 2002), p. 479.

${ }^{31}$ Y. K. Watanabe and H. Mizunuma, "Slip of Newtonian fluids at solid boundary," JSME Int. J., Ser. B 41, 525 (1998).

${ }^{32}$ R. Pit, H. Hervet, and L. Leger, "Direct experimental evidence of slip in hexadecane: solid interfaces," Phys. Rev. Lett. 85 980 (2000).

${ }^{33}$ E. Bonaccurso, M. Kappl, and H. J. Butt, "Hydrodynamic force measurements: boundary slip of hydrophobic surfaces and electrokinetic effects," Phys. Rev. Lett. 88, 076103 (2002).

${ }^{34}$ O. I. Vinogradova, "Slippage of water over hydrophobic surfaces," Int. J. Miner. Process. 56, 31 (1999).

${ }^{35}$ J. M. Gersting, "Hydrodynamic stability of plane porous slip flow," Phys. Fluids 17, 2126 (1974).

${ }^{36}$ A. Spille and H. B. A. Rauh, "Critical curves of plane Poiseuille flow with slip boundary conditions," Nonlinear Phenom. Complex Syst. 3, 171 (2000)

${ }^{37}$ C. J. Gan and Z. N. Wu, "Short-wave instability due to wall slip and numerical observation of wall-slip instability for microchannel flows," J. Fluid Mech. 550, 289 (2006).

${ }^{38}$ E. Lauga and C. Cossu, "A note on the stability of slip channel flows," Phys. Fluids 17, 088106 (2005).

${ }^{39}$ Y. X. Zhu and S. Granick, "Limits of hydrodynamic no-slip boundary condition," Phys. Rev. Lett. 88, 106102 (2002).

${ }^{40}$ R. Ling, C. Jian-Guo, and Z. Ke-Qin, "Dual role of wall slip on linear stability of plane Poiseuille flow," Chin. Phys. Lett. 25, 601 (2008).

${ }^{41}$ A. Beskok and G. E. Karniadkis, Micro Flows Fundamentals and Simulation (Springer, London, 2002).

${ }^{42}$ K. C. Sahu, A. Sameen, and R. Govindarajan, "The relative roles of divergence and velocity slip in the stability of plane channel flow," Eur. Phys. J.: Appl. Phys. 44, 101 (2008).

${ }^{43}$ J. C. Maxwell, "On stresses in varefied gases arising from inequalities of temperature," Philos. Trans. R. Soc. London 170, 231 (1879).

${ }^{44}$ T. Min and J. Kim, "Effects of hydrophobic surface on stability and transition," Phys. Fluids 17, 108106 (2005).

${ }^{45}$ A. K. H. Chu, "Instability of Navier slip flow of liquids," C. R. Mec. 332, 895 (2004).

${ }^{46}$ X. Y. You and J. R. Zheng, "Stability of liquid-liquid stratified microchannel flow under the effects of boundary slip," Int. J. Chem. React. Eng. 7, A85 (2009).

${ }^{47}$ M. Webber, "Instability of fluid flows, including boundary slip," Doctoral thesis (Durham University, 2007), Available at Durham E-Theses Online: http://etheses.dur.ac.uk/2308/.

${ }^{48}$ K. C. Sahu and R. Govindarajan, "Linear stability of double-diffusive two-fluid channel flow," J. Fluid Mech. 687, 529 (2011).

${ }^{49}$ E. Lauga, M. P. Brenner, and H. A. Stone, in Handbook of Experimental Fluid Dynamics, edited by J. F. Foss, C. Tropea, and A. Yarin (Springer, New York, 2005).

${ }^{50}$ P. G. Drazin and W. H. Reid, Hydrodynamic Stability (Cambridge University Press, Cambridge, 1985).

${ }^{51}$ C. Canuto, M. Y. Hussaini, A. Quarteroni, and T. A. Zang, Spectral Methods in Fluid Dynamics, 1st ed. (Springer Verlag, New York, 1987).

${ }^{52}$ R. Usha, O. Tammisola, and R. Govindarajan, "Linear stability of miscible two-fluid flow down an incline," Phys. Fluids 25, 104102 (2013). 